\title{
1 Somatic Mutations in Clonally Expanded T-lymphocytes in Patients with Chronic Graft-Versus-Host
} 2 Disease

Giljun Park ${ }^{1,2}$, Daehong Kim ${ }^{1,2}$, Jani Huuhtanen ${ }^{1,2}$, Sofie Lundgren ${ }^{1,2}$, Rajiv K. Khajuria ${ }^{1}$, Ana M. Hurtado ${ }^{3}$, Cecilia Muñoz-Calleja ${ }^{4}$, Laura Cardeñoso ${ }^{5}$, Valle Gómez-García de Soria ${ }^{6}$, Tzu Hua Chen-Liang ${ }^{3}$, Samuli

7 Eldfors ${ }^{7}$, Pekka Ellonen ${ }^{7}$, Sari Hannula ${ }^{7}$, Oscar Bruck ${ }^{1,2}$, Anna Kreutzman ${ }^{1,2}$, Urpu Salmenniemi ${ }^{8}$, Tapio

8 Lönnberg $^{9}$, Andres Jerez ${ }^{3}$, Maija Itälä-Remes ${ }^{8}$, Mikko A. I. Keränen ${ }^{1,2,10}$, and Satu Mustjoki, MD ${ }^{1,2}$

${ }^{1}$ Hematology Research Unit Helsinki, Helsinki University Hospital Comprehensive Cancer Center, Helsinki, Finland ${ }^{2}$ Translational Immunology Research Program and Department of Clinical Chemistry and Hematology, University of Helsinki, Helsinki, Finland

${ }^{3}$ Hematology and Medical Oncology Department, Hospital Morales Meseguer. Centro Regional de Hemodonación. Universidad de Murcia. IMIB, MURCIA, Spain

${ }^{4}$ Department of Immunology, Hospital Universitario de La Princesa, Instituto de Investigación Sanitaria Princesa, Madrid, Spain

${ }^{5}$ Department of Microbiology, Hospital Universitario de La Princesa, Instituto de Investigación Sanitaria Princesa, Madrid, Spain

${ }^{6}$ Department of Hematology, Hospital Universitario de La Princesa, Instituto de Investigación Sanitaria Princesa, Madrid, Spain

${ }^{7}$ Institute of Molecular Medicine Finland (FIMM), University of Helsinki, Helsinki, Finland

${ }^{8}$ Turku University Hospital, Stem cell transplantation unit, Turku, Finland

${ }^{9}$ Turku Centre for Biotechnology, University of Turku and Åbo Akademi University, Turku, Finland

${ }^{10}$ Department of Hematology, Helsinki University Hospital Comprehensive Cancer Center, Helsinki, Finland

\section{Corresponding author:}

Prof. Satu Mustjoki

Hematology research unit Helsinki,

University of Helsinki and

Helsinki University Hospital Comprehensive Cancer Center

P.O. Box 700, Haartmaninkatu 8

FIN-00029 Helsinki, Finland

e-mail: satu.mustjoki@helsinki.fi

Tel +3589471 71898, fax +358947171897 


\section{ABSTRACT}

39 Graft-versus-host-disease (GvHD) is the main complication of allogeneic hematopoietic stem cell

40 transplantation. GVHD patients have aberrant T cell expansions, which are thought to drive pathological

41 immune activation. Here we report mechanistic insights that somatic mutations may account for persistent

42 clonal T cell expansions in chronic GvHD (cGvHD). In an index patient suffering from cGVHD, we discovered

43 persisting somatic MTOR, NFKB2, and TLR2 mutations in an expanded CD4+ T clone. In the screening

44 cohort ( $\mathrm{n}=135)$, the MTOR P2229R kinase domain mutation was detected in two additional cGvHD patients,

45 but not in controls. Functional analysis of the discovered MTOR mutation indicated a gain-of-function

46 alteration in translational regulation yielding in up-regulation of phosphorylated S6K1, S6, and AKT. Paired

47 single-cell RNA and T cell receptor alpha and beta sequencing strongly supported cytotoxicity and

48 abnormal proliferation of the clonally expanded CD4+ T cells. Real-time impedance measurements

49 indicated increased cytotoxicity of mutated CD4 + T cells against the patient's fibroblasts. High throughput

50 drug-sensitivity testing suggested that mutations induce resistance to mTOR inhibitors but increase

51 sensitivity for HSP90 inhibitors. Our findings suggest a novel explanation for the aberrant, persistent T cell

52 activation in $\mathrm{CGvHD}$, and pave the way for novel targeted therapies. 


\section{INTRODUCTION}

55

Graft-versus host disease (GvHD) is the main complication of allogeneic hematopoietic stem cell transplantation (allo-HSCT).(1) Chronic GvHD (cGvHD), that occurs more than 100 days after the transplantation, develops in $30-70 \%$ of allo-HSCT recipients. Affected patients frequently need immunosuppressive treatment for years or even for a lifetime, and in many patients the condition is fatal.(2) The genesis of cGvHD is multifactorial, but donor alloreactive lymphocytes are believed to be the key pathogenetic drivers that target host tissues such as skin, soft tissues, oral mucosa, and eyes. In particular, CD4+ T cells contribute to the early inflammation and tissue injury, to subsequent chronic inflammation, and late aberrant tissue repair and fibrosis.(3)

During normal immune response, naïve T cells encounter their cognate antigen, get activated and undergo a rapid clonal expansion.(4) The activation and proliferation of T cells are usually tightly regulated processes, in which effector cells undergo apoptosis upon proper immune response. In many immunesystem-mediated disorders, such as cGvHD, the immune homeostasis is disturbed, and the enormous variability of different T cell clones is diminished. In some patients, the T cell receptor (TCR) repertoire is heavily skewed, and clones comprising up to $20-40 \%$ of all T cells can exist.(5) The underlying mechanisms for this phenomenon remain unknown.

Here, we hypothesized that in cGvHD antigen-encountered T cells may acquire somatic mutations due to constant immune-system activation and proliferation. Such mutations might lead to functional and survival advantages of T cells that result in clonal expansion and aberrant immune responses. To explore this theory, we sequenced purified CD4+ and CD8+ lymphocytes from an index patient suffering from cGVHD with a custom deep-sequencing panel consisting of immunity and inflammation-related genes. Mutation findings were confirmed in a validation cohort of 135 GVHD patients. Subsequently, the functional consequences of the discovered mutations including their role in conferring resistance to 
78 immunosuppressive therapy were evaluated in vitro, and finally verified with patient cells using drug

79 sensitivity screening and unbiased transcriptome-wide single-cell RNA-sequencing (scRNA-seq) paired with

80 T cell receptor alpha and beta sequencing (TCRab-seq) analysis and functional cytotoxicity assays.

81 


\section{Clinical Characteristics of the Index cGvHD Patient}

The index patient was a 56-year-old male, who was diagnosed with chronic phase chronic myeloid leukemia in 1999. The clinical status and treatment history are described in detail in Supplemental Figure S1 and Supplemental results. Since the beginning of 2001, the patient suffered from cGvHD affecting his liver, eyes, nails, and skin. The immunosuppression was continuously adjusted according to the clinical presentation of the cGvHD.

\section{Immunophenotype and clonal expansions of CD4+ and CD8+ T cells}

During the first sampling in 2013 , the patient was on mycophenolate mofetil therapy. T cell clonality was

initially analyzed with a flow cytometry-based assay using a panel of TCR V $\beta$-specific antibodies. A large clonal V $\beta 20+$ expansion was noted among CD4+ T cells. The V $\beta 20+$ clone constituted approximately $50 \%$ of pure $\mathrm{CD} 4+\mathrm{T}$ cells and $60 \% \mathrm{CD} 4+\mathrm{CD} 8+\mathrm{T}$ cells (Figure $1 \mathrm{~A}$ and $1 \mathrm{~B}$ ). Additionally, smaller clonal expansions (Vß5.1 11.8\%, V 37.1 21.0\%, V 317 12.1\%, and V 323 12.2\%) were detected among CD8+ T cells (Figure 1B). To assess the clonality in more detail, FACS-sorted CD4+ V $\beta 20+, C D 4+V \beta 20$ - and CD8+ T cells, obtained in 2015, were further analyzed by a TCR $\beta$ deep-sequencing assay(6), which confirmed the TCRBV30-01 clone expansion (corresponding to the $V \beta 20+$ expansion observed by flow cytometry) in the $C D 4+V \beta 20+$ fraction (66.2\% of sorted cells) (Figure 1 C). TCRß sequencing of CD8+ T cells revealed two relatively large clones, TCRBV07-09 (16.1\%) and TCRBV28-01 (17.9\%).

101 During an exacerbation of sclerodermatous skin lesions in $2015,59 \%$ of peripheral blood leukocytes were T

102 cells, 5\% B cells, and 35\% NK cells (Figure S2A in the Supplementary Appendix). CD3+ T cells were composed of CD4+ (47\%), CD4+CD8+ (17\%), and CD8+ T cells (27\%) (Figure S2 in the Supplementary Appendix). An increased number of CD4+ effector memory (EM, 75.0\%) and terminally differentiated 
106

memory (CM) cells (6.2\%) when compared with the sibling donor's CD4+ T cell pool (59.6\% EM, 5.0\%

TEMRA, and 19.9\% CM cells)(Figure 1D). In the CD8+ T cell pool, increased amount of TEMRA cells was noted $(79.9 \%$ of CD8+ T cells).

\section{Somatic Mutations in the Expanded CD4+ T Cell Population of the Index Patient}

To screen for somatic mutations, a customized immunity and inflammation-related gene sequencing panel (immunogene panel)(6) was applied to immunomagnetic bead-separated blood CD4+ and CD8+ T cells, that were obtained from the index patient in 2013. The median target gene coverage for the panel was 152 in CD4+ and 160 for CD8+ T cells. In total, 14 candidate putative somatic mutations were discovered within the CD4+ T cells (Table 1), and one in CD8+ cells (Table S7A in the Supplementary Appendix). Based on the known biological significance, three of the mutations (MTOR, NFKB2, and TLR2) were considered as putative driver mutations and potentially important for disease pathogenesis and were studied further. The previously undescribed somatic missense mutation in MTOR (position 11182160, G to C) changes the amino acid proline 2229 to arginine (Figure 2A). The variant allele frequency (VAF) was $13.3 \%$ among CD4+T cells (Table 1). This mutation in exon 48 is located in the kinase domain which has been suggested to be important for signal transduction.(7) In addition to MTOR, two other interesting mutations were identified in the NFKB2 and TLR2 genes, although these were statistically not significant $(p>0.01)$ due to the low coverage in these locations (Table 1). The NFkB2 missense mutation (position 104162075, C to A) leads to a change of the amino acid proline 882 to glutamine (Figure 2A). TLR2 missense mutation (position 154625732, G to T) results in a change of the amino acid tryptophan 558 to leucine (Figure 2A). The transcription factor NFkB2 is a critical regulator of inflammation and immune function.(8) Toll-like-receptor 2 (TLR2) is one of the pattern recognition receptors and has been shown to be a crucial player for the pathogenesis of autoimmune diseases. Notably, TLR2 protein has been shown to be highly expressed in GvHD patients.(9) 
As an additional confirmation of these findings, we performed exome sequencing of CD4+, CD8+ T cells, and NK-cells obtained from the index patient in 2015 (Tables S7B, C, and D in the Supplementary Appendix). Altogether, 15 candidate putative somatic mutations were discovered within the CD4+ T cell population, including those in the MTOR, TLR2, and NFkB2 genes (Table S7 in the Supplementary Appendix).

\section{Validation of the MTOR, TLR2, and NFkB2 Mutations in the Index Patient}

To further validate the MTOR P2229R, TLR2 W558L, and NFkB2 P882Q mutations, CD4+ cells obtained in 2015 underwent standard capillary sequencing. Only the MTOR mutation was confirmed due to the low sensitivity of the assay (Figure 2B). Therefore, targeted amplicon sequencing with a coverage up to 100.000X and a sensitivity of $0.5 \% \operatorname{VAF}(10)$ was applied to all available samples from different time points to establish the dynamics and the lineage specificity of the discovered mutations. The MTOR mutation was detected in CD4+ T cells that were obtained in 2015 and 2017 with VAFs $19.2 \%$ and $21.0 \%$, respectively (Table 2 and Table S7 in the Supplementary Appendix). The VAF of the MTOR mutation appeared to increase from 2013 to 2017 regardless of the continuous immunosuppressive therapy (Figure 2C). No mutations or very low VAFs were detected within flow-sorted CD3-, CD8+/CD4-, CD4+Vß20-, CD4+CD8+ V $320-$, and monocyte samples (Figure S2C and Table S8 in the Supplementary Appendix). Thus, the mutation was confined to the CD4+ V $320+$ cell fraction (VAF $44.7 \%$ in flow-sorted cells).

Similarly, the NFkB2 P882Q mutation was confirmed to be limited to CD4+V $\beta 20+T$ cells (VAFs: $12.0 \%$ in CD4+ T cells and 21.3\% in CD4+Vß20+ cells) (Table 2, S7 and S8 in the Supplementary Appendix). In contrast, TLR2 W558L mutation was discovered in both CD4+ (VAFs: $16.5 \%$ in CD4+ cells, $35.5 \%$ in CD4+VB20+ cells) and CD8+ T cells (VAF $4.7 \%$ ) (Table S9 and S10 in Supplementary Appendix). 
153

In the course of the disease, the cGvHD affected different organs of the index patient, but particularly the skin. To explore whether lymphocytes harboring the detected somatic mutations can be found in target organs, we screened paraffin-embedded biopsy samples by amplicon sequencing. The MTOR mutation was identified in a sclerodermatous lesion that was biopsied in 2015 (VAF $0.8 \%$ ), but not in eye or liver biopsies (Table 2). Immunofluorescence staining demonstrated CD4+ and CD8+ T cell infiltration in the same sclerodermatous lesion (Figure 2D).

To examine whether the mutations were already present in the donor, both CD4+ and CD8+ T cells from the donor were sequenced by amplicon sequencing, but no mutations were detected.

\section{Screening for the Identified MTOR, TLR2, and NFkB2 Mutations in CGvHD patient Cohort}

To explore whether the found mutations are recurrent, blood samples from 135 cGvHD patients, 38 alloHSCT patients without cGvHD, and 54 healthy controls were screened by the amplicon sequencing. Two additional cGvHD patients carried the same MTOR missense mutation yielding in a MTOR P2229R mutation frequency of $2.2 \%$ in all cGvHD patients (3 out of 135). In healthy controls or allo-HSCT patients without cGvHD, no MTOR mutations were detected. Furthermore, NFkB2 mutations were neither detected in additional cGvHD patients nor in controls. The TLR2 W558L mutation was found in both CGvHD patients and healthy controls, but the VAF indicated a 10- and 4-fold higher mutation frequency in cGvHD patients' CD4+ and CD8+ T cells compared to healthy controls (Figure S3, Tables S9 and S10 in the Supplementary Appendix).

\section{Identified Somatic Mutations Result in a Gain-of-function Alteration}

MTOR consists of two functionally distinct multi-protein complexes, mTORC1 and mTORC2. Eukaryotic translation initiation factor 4E (elF4E)-binding protein 1 (4E-BP1) and ribosomal S6 kinase (S6K1) are among the key substrates of mTORC1, and as such critical regulators of cap-dependent translation.(11) mTORC2 
directly phosphorylates AKT, thereby promoting cell survival.(12) Various genomic alterations have been shown to aberrantly activate the mTOR pathways(13), which is marked by an increased phosphorylation of downstream factors, such as S6K1, S6, and AKT. To examine the functional consequences of the MTOR $P 2229 R$ and other mutations, we transduced HEK293 human embryonic kidney cells with mutant constructs. Both the MTOR P2229R single mutant and triple mutant resulted in a substantially enhanced phosphorylation of S6K1, S6, and AKT compared to wild-type (WT) MTOR or triple WT (Figures 3A and B) suggesting an activation of both mTORC1 and mTORC2 pathways.

The NFkB2 P882Q mutation is located in the c-terminal domain (Figure 2A), which is known to play an important role in the ubiquitination and partial proteolysis from NFkB2 (p100) to NFkB2 (p52).(14) In order to determine the molecular balance between these two states, we performed immunoblotting which revealed an increased expression of p52 (Figures $3 C$ and D) indicating a gain-of-function alteration. Similarly, to study the functional consequences of the TLR2 W558L mutation, we evaluated alterations in transcriptional regulation by analyzing mRNA expression levels of a subset of TLR2 downstream targets by qRT-PCR. This demonstrated significantly increased expression levels of ELK1 and FOS1 in the TLR2 W558L mutant expressing cell line as compared to the WT control (Figure S4B in the Supplementary Appendix).

\section{Paired scRNA- and TCRab sequencing of the CD4+ $T$ cells from the index patient from two time points}

Recently, entire transcriptome at the single-cell level has been explosively studied for revealing a differential gene expression profiles between individual cells, which cannot be identified from analysis of mixed cells.(15)

In order to better understand the heterogeneity of the clonotype and the underlying CD4+ T cell compartment in an unbiased manner, we performed simultaneous single-cell RNA and paired TCRab sequencing on two time points in 2015 and 2017 for the index patient's CD4+ T lymphocytes from peripheral blood. From the paired sequencing we received 15,847 CD4+ T lymphocytes passing the quality 
control, and they could be divided into nine distinct phenotypes with graph-based clustering. Interestingly, most of the cells (72.0\%) were characterized by cytotoxicity (clusters $0,1,2$ and 3 ), and lower frequency of naïve cells (20.8\%, clusters 4 and 5$)$ and regulatory T cells (3.2\%, cluster 8 ) were identified (Figure 4 A-C).

The frequency of cells in clusters were stable between the time points indicating resistance to the ongoing immunosuppressive treatment, and only the two smallest populations showed over two-fold-change between the two timepoints (clusters 3 and 7, Figure $S 5$ in the Supplementary Appendix).

From the TCRab-seq we detected TCRab, TCRa or TCRb from 11,055 cells (71.4\%), resulting in 3651 unique T cell clonotypes. The clonotype matching to TCRVB sequencing data (Figure 1C) and harboring the MTORmutation was also the most expanded fraction, representing $40.1 \%$ of the CD4+ T cells. Almost all of the cells from this clonotype (90.1\%) belonged to the cytotoxic clusters, and most of the cells were included in the cluster 0 (74.6\%) (Figure 4D).

212 To understand the effect of the MTOR-mutation on the T cells, we performed differential expression (DE) analysis between the cytotoxic cells, comparing the cells from clonotype of interest against the other cytotoxic cells in clusters $0,1,2$ and 3 . The analysis found 876 statistically significant DE-genes, of which 694 were upregulated in the clonotype, including cytotoxic genes (e.g. GZMA, GZMAB, GNLY, GZMK, NKG7 and PRF1), and HLA class I and II genes (Figure 4E). Additionally, upregulated eukaryote elongation factors

217 (eEFs), such as EEF1A1, EEF1B2, and EEF2, supported abnormal growth and proliferation of the expanded 218 CD4+ T cells. Furthermore, the expression of DUSP2 and KLRB1 genes was highly specific for the mutated 219 clone (Figure 4B). To identify differential pathway regulation in the clonotype, Gene Set Enrichment 220 Analysis (GSEA) was performed, resulting in 11 significantly over-represented and 0 under-represented pathways in the clonotype (Table S11 in the Supplementary Appendix). The upregulated pathways included MTORC1-pathway supporting the similar effect of the mutations in patient cells as observed in the in vitro response and IL2-STAT5 signaling. 


\section{Real-time cytotoxicity analysis of CD4+ T cells against primary fibroblasts from the index patient}

Real-time electrical impedance measurements monitoring target cell killing have been widely applied to study the cellular cytotoxicity in vitro. $(16,17)$ To test the functional effects of the mutated CD4+ T cells and to verify aberrantly upregulated gene expression signatures associated with cytotoxicity, we performed coculture experiments with CD4+ T cells and primary fibroblasts. Addition of purified CD4+ $\mathrm{T}$ cells on the mono-layer of primary fibroblasts from the index patient resulted in decreased electrical impedance implicating cytotoxicity of the CD4+ T cells (Figure 5A and B). In contrast, the CD8+ T cells showed no cytotoxic activity against the fibroblasts, as the impedance curve mirrored the control well without effector cells.

\section{Drug Sensitivity and Resistance Testing (DSRT) in CD4+ T cells from the Index Patient}

To determine sensitivity of the mutated cells to targeted therapy, robust ex vivo DSRT with 527 drugs in 5 different concentrations (18) was performed on freshly isolated CD4+ T cells from the index patient, the donor, and a healthy control (Figure 6). In this screen, the index patient's CD4+ T cells were less sensitive to mTOR/PI3K inhibitors as compared to the donor's CD4+ T cells (Figures 6A and B), although constitutive $\mathrm{PI3K/AKT/mTOR} \mathrm{activity} \mathrm{generally} \mathrm{predicts} \mathrm{rapalog} \mathrm{sensitivity.} \mathrm{Instead,} \mathrm{we} \mathrm{observed} \mathrm{that} \mathrm{heat} \mathrm{shock}$ protein 90 (HSP90) inhibitors showed an increased killing effect on the index patient's CD4+ T cells as compared to CD4+ T cells from the donor and healthy control (Figures 6A, C, and D). Interestingly, scRNAseq analysis indicated that one of HSP90 family members, HSP9OAB1, was significantly upregulated in the expanded CD4+ T cell clonotype, and it was one of the gene expression markers for the clonotype (Figure $4 \mathrm{E})$. With regard to other clinically interesting drug classes, both the donor and the recipient CD4+ T cells were sensitive to HDAC inhibitors, CDK-inhibitors, and proteosome inhibitors. The donor cells were also modestly more sensitive to glucocorticoids (dexamethasone and methylprednisone), but this was not statistically significant. Neither donor nor recipient CD4+ T cells were sensitive to cyclophosphamide, 
249 tacrolimus, or methotrexate. However, it should be taken into account that the assay read-out is cell death,

250 and lymphocytes were not activated nor actively proliferating during the experiment.

251 In both WT and mutant HEK293 cells, the HSP90 inhibitor ganetespib reduced AKT phosphorylation on

252 serine 473. As expected, AKT-phosphorylation appeared to be normal following sirolimus treatment as

253 rapalogs only inhibit mTORC1 activity (Figures $6 \mathrm{E}$ and F). Treatment with either drug resulted in decreased

254 levels of phosphorylated S6K1 in both mutant and WT cells (Figure 6G). Both drugs also led to a reduced

255 pS6 phosphorylation in CD4+ T-lymphocytes from the index patient and a healthy control (Figures $6 \mathrm{H}$ and

256 I), suggesting an inhibitory effect on mTORC1 activity. Likewise, the scRNA-seq data analysis supported that

257 MTORC1 pathway is upregulated in the clonally expanded cytotoxic CD4+ T cells from the index patient

258 (Table S11 in the Supplementary Appendix). AKT was more phosphorylated in the patient's CD4+ T cells as compared to controls with a slight decrease in both samples upon treatment with ganetespib or sirolimus.

\section{Discussion}

By analyzing blood CD4+ and CD8+ T cells we discovered a recurrent somatic missense mutation in MTOR in cGvHD patients. In the index patient, the mutation was limited to the expanded CD4+ T cell clone, it persisted for years and was found in both blood and sclerodermatous skin lesion samples. Paired scRNAand TCRab-seq verified that the majority of the expanded CD4+ T cells had upregulated expression of genes associated with cytotoxicity and cellular proliferation. Furthermore, mutated CD4+ T cells possessed cytotoxicity against patient's own primary fibroblasts. No mutations were discovered in the sibling donor samples suggesting the mutations been formed after the allo-HSCT. Functional in-vitro studies indicate that the MTOR mutation results in a gain-of-function alteration activating both mTORC1 and mTORC2 pathways. Accumulation of somatic mutations is inherently associated with normal cell division. The role of somatic mutations in cancer is well established, and interestingly, recent reports suggest that somatic 
shown that somatic mutations occur in cytotoxic lymphocytes of newly diagnosed rheumatoid arthritis patients(6) and in patients with large granular lymphocytic proliferation.(24) Interestingly, it was also recently shown, that the disruption of the TET2 gene by lentiviral vector-mediated insertion of the chimeric antigen receptor (CAR) transgene led to the expansion of single CAR T cell in a patient with chronic lymphocyte leukemia and enhanced therapeutic efficacy.(25) In addition to lymphoid cell-related disorders, recent studies have suggested that somatic mutations may play a role in the pathogenesis of other noncancerous diseases such as KRAS mutations in brain arteriovenous malformations leading to activation of the MAPK-ERK pathway.(23)

The novel MTOR P2229R kinase domain mutation discovered in our cohort has not been previously reported, although already over 750 different MTOR mutations exist in the COSMIC database. Previously, mutations in the same domain have been shown to lead to the activation of the mTORC1. The $\mathrm{PI3K} / \mathrm{AKT} / \mathrm{mTOR}$ axis controls important cellular processes and is frequently dysregulated in various cancer types.(26) This pathway is also important for the regulation of T cell activation, function, and survival.(27) Inhibition of the PI3K/AKT/mTOR axis by targeting the mTORC1 complex with rapamycin has been used to prevent and treat GvHD.(28) Based on our data, the MTOR P2229R mutation induces the activation of both the mTORC1 and mTORC2 as noted by increased S6K and AKT phosphorylation. Additionally, scRNA-seq analysis strongly supported that MTORC1 pathway is upregulated in the clonally expanded and cytotoxic CD4+ T cells from the index patient.

In addition to MTOR, the mutation in the NFKB2 gene is also a putative driver due to the importance of this signaling pathway in the immune system. NF-KB has been extensively described as one of the main regulators of the inflammatory response and cancer pathogenesis, offering a promising target in anti-inflammation and -cancer drug development.(29) Both p50/RelA-mediated canonical and p52/RelBmediated non-canonical pathways are involved in NFkB activation. Phosphorylated NFkB2 (p100) is associated with RelB and sequentially ubiquitinated at the C-terminus to form the p52/RelB complex, 
which is translocating into nucleus and activating downstream target genes.(30) Therefore, p52 formation in NFkB2 (p100) processing is a key step for the activation of the non-canonical NFkB pathway. Especially two serine residues in the C-terminal domain of NFkB2 (p100), S866 and S870, are necessary for NFkB2 (p100) processing.(31) The index patient harbored NFkB2 (p100) P882Q somatic mutation located in the Cterminus, which has not been reported in the COSMIC before. This mutation leads to an increased p52 formation which potentially induces a hyper-activation of non-canonical NF-KB pathway contributing to chronic inflammation in the index patient.

Unlike the discovered NFKB2 and MTOR mutations, the TLR2 W558L somatic mutation was identified in both cGvHD patients and healthy controls. However, VAFs were significantly higher in cGvHD patients as in healthy controls. TLR2 is one of the pathogen-associated molecular pattern recognition receptors and regulators of innate immunity, and it is constitutively expressed on regulatory and memory CD4+ T cells.(32) TCR activation by an anti-CD3 antibody has been shown to induce the overexpression of TLR2 on naïve CD4+ T cells followed by activation of the MyD88-dependent NF-KB signaling cascade and inflammatory gene expression in peripheral blood.(33) Thus, in combination with the NFkB2 and MTOR mutations, the TLR2 W558L mutation may further enhance the activation of the NF-KB and inflammatory pathways.

Single-cell RNA-seq analysis revealed that the clonally expanded CD4+ T cells had upregulated gene expression signatures associated with cytotoxicity (GZMA, GZMB, GNLY and NKG7) and proliferation (DUSP2, KLRB1, EEF1A1, EEF1B2, and EEF2). Furthermore, real-time impedance analysis indicated cytotoxicity of $\mathrm{CD} 4+\mathrm{T}$ cells from the index patient, whereas the CD8+ T cells had no effect. Although cytotoxicity has not been considered as a typical CD4+ T cell function (34), cytotoxic potential of T helper cells (CD4+CTL) has been recently described including a granzyme-mediated killing capability of target cells during viral infections. $(35,36)$ Epigenetic and molecular mechanisms leading into CD4+CTL differentiation has not yet been clearly described.(37) From the proliferation associated genes, DUSP2 (PAC-1) was one of 
the hallmark genes associated with the mutated clonotype. It is known as one of the MAPK phosphatases, which play an important role in deactivating MAPK. DUSP2 is shown to be up-regulated in T cell activation associated with inflammation.(38) Murine knockout phenotype studies for DUSP2 presented reduced cytokine production and protected from inflammatory arthritis. In the murine colitis model, DUSP2 knockout induced Th17 differentiation by directly enhancing the transcriptional activity of STAT3. Therefore, DUSP2 inhibit Th17 lineage of T cell development by attenuated STAT3 activity through dephosphorylation of STAT3 at Tyr705 and Ser727.(39) In addition, it has been indicated that DUSP2 inhibit JNK leading to ERK pathway activation.(40) Interestingly, TLR2 stimulation also strongly correlate with ERK activation. ERK(1/2) pathway is well known for a positive selection of T cell development and proliferation, and especially CD4+ T cell differentiation depends on ERK signaling.(41) Taken together, DUSP2 upregulation in scRNA seq data support the expansion of CD4+T cells in the index cGVHD patient. Drug sensitivity testing with patient cells indicated a lower efficacy of mTOR/PI3K inhibitors compared to the donor, suggesting a hyperactivation of mTOR pathway due to the mutation. However, the effect of ongoing immunosuppressive treatment (mycophenolate) cannot be ruled out as it may also affect mTOR signaling.(42) Since the mTOR pathway is hyperactivated in many different types of cancer and autoimmune diseases, mTOR inhibitors have been developed and applied to prevent dysregulated mTOR signaling.(43-46) Rapamycin and its analogs are highly specific mTOR inhibitors that form a complex with FKBP2, which selectively binds to the FRB domain of mTOR, leading to targeted inhibition of mTORC1mediated signaling pathways. Therefore, alternative therapeutic applications have been suggested for additionally targeting the dysregulated mTORC2 signaling pathway. Initially, Zheng et al. indicated that the kinase domain of mTOR is a more potent site for mTOR inhibition as it is necessary for both rapamycininsensitive and rapamycin-sensitive aspects of cell growth and survival.(47) ATP-competitive mTOR inhibitors targeting the kinase domain of mTOR have already been developed to inhibit both mTORC1 and 
mTORC2-mediated signaling processes. $(48,49)$ These inhibitors demonstrated a better clinical efficacy and

lower toxicity in anti-tumor therapy as compared with rapalogs.(7)

Interestingly, HSP9O inhibitors showed higher efficacy when compared to mTOR inhibitors in the

DSRT assay. HSP90 is one of the most abundant and conserved ATP-dependent molecular chaperons, whose expression increases by up to 10 -fold under physiologic stress conditions.(50) Functionally, HSP90 plays an important role in the refolding of denatured proteins under stress conditions. $(51,52)$ In addition, it activates these proteins including growth-stimulating proteins and kinases.(50) mTOR, through its mTORC1 component raptor, directly binds to HSP90 in primary T cells in order to regulate mTOR signaling processes.(53) Additionally, AKT physiologically interacts with HSP90 to activate mTOR pathway signaling.(54) Notably, HSP90 overexpression has been suggested to be correlated with Akt/mTOR pathway activation in cancer. Furthermore, HSP90 inhibitors have been shown to suppress the Akt/mTOR pathway activity.(55) Therefore, HSP90 has become a therapeutic target for several cancer types, and many HSP90 inhibitors are under the evaluation in phase I and II clinical trials for cancer therapy.(56) Importantly, HSP90 inhibitors have also been suggested to have protective and therapeutic effects in mouse models of GvHD.(57) To our knowledge, these have not yet been used to treat GvHD patients, but based on our results HSP90 inhibitors could serve as a novel therapeutic approach in a subset of cGvHD patients. In conclusion, novel MTOR, NFkB2, and TLR2 somatic mutations were discovered in an expanded CD4+ T cell clone in a patient with cGvHD. The mutations persisted over time and induced activation of the NF-KB and MTOR pathways. The MTOR mutation was found to be recurrent in other cGvHD patients. Although the MTOR mutation frequency was low in the total cGvHD cohort, somatic mutations may also exist in other genes, and similar small subgroups of cGvHD patients can be discovered warranting further investigations. Our findings imply a novel mechanism for the aberrant, persistent T cell activation in cGvHD and pave the way for potential novel individualized therapies. 


\section{Study Patients}

Samples were collected between 2007-2016 from 135 patients who had developed cGvHD after allo-HSCT

(Helsinki University Hospital, Helsinki, Finland, n=8; Turku University Hospital, Turku, Finland, n=37;

Hospital de la Princesa, Madrid, Spain, n=19; Hospital Morales Meseguer, Murcia, Spain, n=71). In addition,

38 patients who had not developed cGvHD until the date of sampling, served as a control cohort (Turku

University Hospital $n=6$ and Hospital Morales Meseguer $n=32$ ). The blood samples were collected 3 to 102

months (mean 13.5 months) and 2 to 47 months (mean 14.5 months) after allo-HSCT for GvHD and non-

cGvHD patients, respectively. Clinical characteristics of the patients are summarized in Supplemental Table

S1. All patients provided written informed consents. Additionally, buffy coat samples from 54 healthy blood patient's sibling donor was also obtained.

The study was performed in compliance with the principles of Helsinki declaration, and was approved by the ethics committees in the Helsinki University Hospital (Helsinki, Finland), Turku University Hospital (Turku, Finland), Hospital de la Princesa (Madrid, Spain) and Hospital Morales Meseguer (Murcia, Spain).

\section{Reagents}

Primary antibodies against NFkB2 (Cat\#: 4882S, Lot: 4), ribosomal protein S6 (Clone: 54D2, Cat\#: 2317S,

Lot\#: 4), phospho-S6 ribosomal protein (Ser235/236) (Clone: D57.2.2E, Cat\#: 4858T, Lot\#: 16), Akt (Clone:

C67E7, Cat\#: 4691T, Lot\#: 20), phospho-Akt (Ser473) (Cat\#: 9271T, Lot\#: 14), p70S6 kinase (S6K) (Clone:

49D7, Cat\#: 2708T, Lot\#: 7), and phospho-p70S6 (S6K) kinase (Thr421/Ser424) (Cat\#: 9204S, Lot\#: 11) were 

or Nanodrop (Thermo Fisher Scientific).

\section{Cell Lines}

\section{Sample Preparation and DNA Extraction}

Lot\#: GR66278-11) was purchased from Abcam. Secondary antibodies, IRDye 700 conjugated anti-mouse and IRDye 800 conjugated anti-rabbit, were purchased from LI-COR Biosciences.

Human embryonic kidney HEK293 cells (Cat\#: CRL-1573, ATCC) and HEK293FT (Cat\#:R70007, Thermo Fisher Scientific) were maintained in high-glucose Dulbecco Modified Eagle medium (Lonza) containing 10\% FBS (Gibco), 1\% penicillin-streptomycin (Invitrogen), and L-glutamine (Lonza) in a $37^{\circ} \mathrm{C}$ humidified incubator with 5\% CO2. Cell line authentication was performed with GenePrint10 System (Promega). HEK293 cell line was authenticated via Promega GenePrint10 System. The result was compared ATCC STR, JCRB STR, ICLC STR database, and DSMZ online STR database. The identity estimates are calculated according to the allele information found in these databases. HEK293FT cell line was derived from Thermo Fisher Scientific with an authentication and only less than 10 passages were used. Mycoplasma test was performed using MycoAlert Mycoplasma Detection Kit (LONZA, Cat\#: LT07-318).

Mononuclear cells (MNCs) were separated from whole blood using Ficoll-Paque ${ }^{\mathrm{TM}}$ PLUS (GE Healthcare). The separated MNCs were then labeled with either CD4+ or CD8+ magnetic beads (Miltenyi Biotec) and sorted by AutoMACs ${ }^{\circledR}$ cell sorter (Miltenyi Biotec) according to the manufacturer's protocol. The purity of sorted fractions was evaluated by flow cytometry and confirmed to be $>98 \%$ (FACsVerse, BD Biosciences). Alternatively, separated MNCs were sorted using FACsAria II (BD Biosciences). Genomic DNA was isolated from fresh or frozen sorted MNCs or from whole blood samples using the Genomic DNA NucleoSpin Tissue kit (Macherey-Nagel). DNA concentration and purity were measured with Qubit2.0 Fluorometer (Invitrogen) 


\section{Flow Cytometry Analysis and Flow-assisted Cell Sorting}

417 For phenotyping of the memory T cell subsets, peripheral blood mononuclear cells (PBMCs) were

418 immunostained with the antibody panel including anti-CD3 PeCy7 (Clone: SK7, Cat\#: 557851, Lot\#:

419 8037645, BD Biosciences), -CD4 PerCP (Clone: SK3, Cat\#: 345770, Lot\#: 6281605, BD Biosciences), -CD8

420 PerCP (Clone: SK1, Cat\#: 345774, Lot\#: 82152, BD Biosciences) -CD45RA Alexa700 (Clone: HI100, Cat\#:

421 560673, Lot\#: 7180940, BD Biosciences), and -CCR7 PE (Clone: 150503, Cat\#: FAB197P, Lot\#: LEU1618031,

422 R\&D System). Stained samples were analyzed with FACSVerse (BD Biosciences) and FlowJo software

423 (Version 10.4.2). For CD4+ T cell TCR Vß20+ clone sorting, PBMCs were immunostained with anti-CD3 APC

424 (Clone: SK7, Cat\#: 345767, Lot\#: 7236657, BD Biosciences), -CD4 PerCP (Clone: SK3, Cat\#: 345770, Lot\#:

6281605, BD Biosciences), -CD8 PE-Cy7 (Clone: SK1, Cat\#: 335822, Lot\#: 8272690, BD Biosciences) and -

Vß20 (IOTest ${ }^{\circledast}$ Beta Mark TCR Vbeta Repertoire Kit, Cat\#: IM3497, Lot\#: 66, Beckman Coulter). Stained cells

were physically isolated by FACs AriallI (BD Biosciences) and analyzed with FlowJo software. Purity of

\section{TCR V $\beta$ Analysis}

TCR V $\beta$ families were analyzed from peripheral whole-blood samples by flow cytometry based antibody

staining using IOTest ${ }^{\circledast}$ Beta Mark TCR V $\beta$ Repertoire Kit (Cat\#: IM3497, Lot\#: 66, Beckman Coulter). Briefly,

CD4+ and CD8+ T cells in whole blood samples were stained with the panel of TCR V $\beta$ antibodies

recognizing 24 members of TCR $\beta$ chain, which covers about $70 \%$ of the normal human TCR V $\beta$ repertoire.

Stained cells were further analyzed using FACSVerse (BD Biosciences).

\section{TCR CDR3 Deep Sequencing}

Isolated genomic DNAs was used for TCRB deep sequencing. Sequencing and data analysis was conducted 


\section{Immunopanel Sequencing}

A customized NGS panel including exonic areas of 986 genes related to immunity and cancer was used to screen for somatic mutations.(6) Genes included in the panel are provided in the Supplemental Table S2. Sequencing was done from both sorted CD4+ and CD8+ T cells. Bioinformatic analysis to identify and annotate somatic variants was performed as previously described.(6, 24)

\section{Validation of the Somatic MTOR Mutation by Capillary Sequencing Analysis}

A specific primer set was designed using the Primer-Blast search (National Center for Biotechnology Information: http://blast.ncbi.nlm.nih.gov/) to validate the somatic MTOR mutation (Supplementary Table S3). Polymerase chain reaction (PCR) products were purified with the ExoSAP-IT (Affymetrix) followed by sequencing on DNA sequencer (Applied Biosystems). Sequences were analyzed using 4Peaks version 1.7.1.

\section{Amplicon Sequencing of MTOR, NFkB2 and TLR2}

Targeted amplicon sequencing was performed with an in-house developed deep amplicon sequencing panel using the Illumina Miseq platform (Supplementary Table S4). The coverage was over $100,000 \mathrm{X}$, and a variant was called if variant base frequency was $0.5 \%$ of all reads covering a given a position. All variants with the base quality frequency ratio (ratio of number of variant calls/ number of all bases and quality sum of variant calls / quality sum of all bases at the position) $\geq 0.9$ were considered as true somatic variants. A detailed sequencing protocol and the bioinformatics pipeline used for data analysis are described in previous reports. $(6,24)$ 
CD4+ T cells from two time points of the index patient were enriched using CD4 microbeads (Miltenyi Biotec). Single cells were partitioned using a Chromium Controller (10x Genomics) and scRNA-seq and TCRab-libraries were prepared using Chromium Single Cell 5' Library \& Gel Bead Kit (10x Genomics), as per manufacturer's instructions (CG000086 Rev D). In brief, 17,000 cells from each sample, suspended in $0.04 \%$ BSA in PBS were loaded on the Chromium Single Cell A Chip. During the run, single-cell barcoded cDNA is generated in nanodroplet partitions. The droplets are subsequently reversed and the remaining steps are performed in bulk. Full length cDNA was amplified using 14 cycles of PCR (Veriti, Applied Biosystems). TCR cDNA was further amplified in a hemi-nested PCR reaction using Chromium Single Cell Human T Cell V(D)J Enrichment Kit (10x Genomics). Finally, the total cDNA and the TCR-enriched cDNA was subjected to fragmentation, end repair and A-tailing, adaptor ligation, and sample index PCR (14 and 9 cycles, respectively). The gene expression libraries were sequenced using an Illumina NovaSeq, S1 flowcell with the following read length configuration: $\operatorname{Read} 1=26, i 7=8, i 5=0$, Read2 $=91$. The TCR-enriched libraries were sequenced using an Illumina HiSeq2500 in Rapid Run mode with the following read length configuration: Read1 $=150, i 7=8, i 5=0$, Read2 $=150$. The raw data was processed using Cell Ranger 2.1.1. with GRCh38 as the reference genome.

During secondary analysis, cells with fewer than 200 or more than 4000 genes, or more than $15 \%$ of the counts from mitochondrially-encoded transcripts were excluded from the analysis. The remaining data was log-normalized and scaled. To reduce the dimensionality of the data, we determined the highly variable genes as the genes with the highest variance-mean ratio. Genes that had mean expression between 0.0125 and 3 on a log-transformed count scale and genes above $0.5 \log ($ variance/mean) were counted as highly variable, resulting in 764 genes. The T-cell receptor V-genes, mitochondrial genes and ribosomal genes $(n=$ 113) were excluded from the results, resulting in a final list of 651 highly variable genes.

Clusters were identified using the graph-based community identification algorithm as implemented in the Seurat-package(28). Prior to calculating cell-cell distances, PCA was performed on the 651 highly variable 
genes on all the QC-positive cells, and the top 50 principal components were kept. To prevent hyperparameter as a function of number of clusters until the first saturation plateau was achieved. The robustness of these clusters was assessed by subsampling cells and doing the analysis iteratively and visually inspecting the results of embedding and differentially expressed genes between the formed clusters. Differential expression analysis was performed based on the t-test, as suggested by Robinson et al(59). Clusters were annotated using canonical cell type markers as well as the differentially expressed genes.

Gene Set Enrichment Analysis (GSEA) (software.broadinstitute.org/gsea/index.jsp) between the clonotype and other cytotoxic cells was performed on genes that were detected at least in $0.1 \%$ of the cytotoxic cells and had at least log fold-change of 0.01 between the clonotype and other cytotoxic CD4+ T cells. The gene list was ordered based on the fold-change. Overlap with HALLMARK-category was assessed and the False Discovery Rate (FDR) calculated while the number of permutations was 1000.

Clonotypes were identified based on the available information, and both total nucleotide level TCRa and TCRb were used if found. Cells for which more than two recombinants were identified were excluded from further analysis. different T cell clonotypes. The clonotype harboring the MTOR-mutation was the most expanded, compromising of 2366 cells (TRA:CLVGDIGNQGGKLIF; TRB:CAWSTGQANNSPLHF). However, we noticed that the second most (TRB:CAWSTGQANNSPLHF, 1545 cells) and third most expanded (TRA:CLVGDIGNQGGKLIF,

509598 cells) clonotypes had only one chain and as they matched to the most expanded clonotype, we treated this as error coming from uncomplete sequencing and pooled the three most expanded clonotypes into one. 


\section{3}

514

515

\section{Analysis of cellular cytotoxicity}

Primary fibroblasts from the index patient were cultured based on previous method.(60) Briefly, skin biopsy from the index patient were dissected in small pieces (approx. $2 \mathrm{~mm} \times 2 \mathrm{~mm}$ ) and transferred into 6-

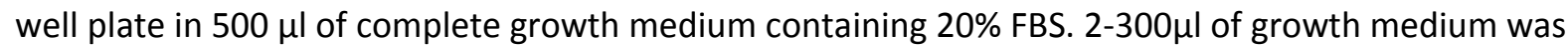
added for every 2 days to replace evaporated media. After one week, increase amount of media to $2 \mathrm{ml}$ and change the media every 3 days. Once cells were confluent in each well, cells were trypsinized and passaged.

To measure cellular cytotoxicity of CD4+ and CD8+ T cells from the index patient, the proliferation of the fibroblast established from the index patient was monitored with XCELLigence ${ }^{\mathrm{TM}}$ real-time cell analyzer (RTCA) (ACEA Biosciences, CA, USA) according to the manufacture's instruction. $x$ CELLigence ${ }^{T M}$ RTCA biosensor measures cellular adhesion through electrical impedance, which is converted to Cell Index (arbitrary units). Briefly, the E-Plate 16 VIEW (ACEA Biosciences, CA, USA) was equilibrated with the $100 \mu l$ of culture media at room temperature. $100 \mu$ l of the cell suspension $\left(8 \times 10^{3}\right.$ cells/well $)$ in duplicate was transferred to the plate followed by incubation at room temperature for $30 \mathrm{~min}$ to allow the cells to settle at the bottom of the wells. The XCELLigenceTM monitored the cells every $30 \mathrm{~min}$ for 200 repetitions. When the cell index [(impedance at time point $\mathrm{n}$-impedance in the absence of cells)/nominal impedance value] were reached a plateau, CD4+ T cells, CD8+ T cells, and NK92 as an cellular cytotoxicity inducer $\left(6.4 \times 10^{4}\right.$ cells as a ratio of the fibroblast to the inducer is $1: 8)$ for the fibroblast were added on the plate. CD4+ T cells and CD8+ T cells were separated from MNCs with CD4+ or CD8+ magnetic beads (Miltenyi Biotec) and sorted by AutoMACs ${ }^{\circledR}$ cell sorter (Miltenyi Biotec) according to the manufacturer's protocol. The real-time impedance trace for the fibroblasts exposed to CD4+ T cells, CD8+ T cells, and NK-92 were monitored for 48 h.

\section{Multiplexed Immunohistochemistry (mIHC)}


537 Tissue blocks were cut in $3.5 \mu \mathrm{m}$ sections. Slides were deparaffinized in xylene and rehydrated in graded

538 ethanol series and $\mathrm{H} 2 \mathrm{O}$. Heat-induced epitope retrieval (HIER) was carried out in $10 \mathrm{mM}$ Tris- $\mathrm{HCl}-1 \mathrm{mM}$

539 EDTA buffer in $+99^{\circ} \mathrm{C}$ for $20 \mathrm{~min}$ (PT Module, Thermo Fisher Scientific). Peroxide activity was blocked in $0.9 \%$

$540 \mathrm{H} 2 \mathrm{O} 2$ solution for $15 \mathrm{~min}$, and protein block performed with 10\% normal goat serum (TBS-NGS) for $15 \mathrm{~min}$.

541 Anti-CD3 (Clone: EP449E, Cat\#: ab52959, Lot\#: GR140731, Abcam) primary antibody diluted 1:500 in

542 protein blocking solution and secondary anti-rabbit horseradish peroxidase-conjugated (HRP) antibodies

543 (Immunologic) diluted 1:1 in washing buffer were applied for $1 \mathrm{~h} 45 \mathrm{~min}$ and $45 \mathrm{~min}$, respectively. Tyramide

544 signal amplification (TSA) 488 (PerkinElmer) was applied on the slides for 10 min. Thereafter, HIER,

545 peroxide and protein block were repeated, followed by application of anti-CD8 (1:500, Clone: C8/144B,

546 Cat\#: BSB 5174, Lot\#: 5174JDL05, BioSB) primary antibody, HRP-conjugated secondary antibody diluted 1:3

547 with washing buffer and TSA 555 (PerkinElmer). HIER, peroxide block and protein block were repeated.

548 Then, the slides were incubated with CD4 primary antibodies (1:25, Clone: EPR6885, Cat\#: ab133616, Lot\#:

549 GR218457, Abcam) overnight in $+4^{\circ} \mathrm{C}$. Next, AlexaFluor647 fluorochrome-conjugated secondary antibody

550 (Thermo Fisher Scientific) diluted in 1:150 and Dapi (Roche) counterstain diluted 1:250 in washing buffer

were applied for $45 \mathrm{~min}$. ProLong Gold mountant (Thermo Fisher Scientific) and a coverslip were applied on

the slides. After peroxide block, antibody incubations and fluorochrome reaction, slides were washed three

Fluorescent images were acquired with the Axiolmager.Z2 (Zeiss) microscope equipped with a Zeiss Plan-

Apochromat 20x objective.

Site-Directed Mutagenesis

Site-directed mutagenesis was conducted using GENEART ${ }^{\circledast}$ Site-Directed mutagenesis system according to 
(GeneCopoeia, Cat.No. EX-Q0161-Lv122, GeneCopoeia), and mTOR (Addgene, Cat.No.26603) expression

vector. The primer sequences used for the site-directed mutagenesis are in the Supplemental Table S5.

Establishing Stable Cell Lines

HEK293 cells were transfected using FuGENE HD transfection reagent (Promega) with either a wildtype or P2229R MTOR expression vector (ratio of reagent to DNA is 3:1) following the manufacturer's instruction. Neomycin resistant clones were selected after the cells were cultured with $\mathrm{G} 418(500 \mu \mathrm{g} / \mathrm{mL})$ for 3 weeks.

The lentiviruses were produced by co-transfection of HEK293FT cells with NFkB2 (wildtype or P882Q mutant) or TLR2 (wildtype or W558L mutant) lentiviral expression vectors, and psPAX2 lentiviral packaging plasmid (Addgene) and pCMV-VSV-G envelope plasmid (Addgene) using Lipofectamine ${ }^{\circledR} 2000$ (Thermo

Fisher Scientific). Antibiotic free DMEM containing 10\% FBS was used as a culturing medium and Opti-MEM replaced with DMEM. After 48 hours, the supernatants were centrifuged at $300 \mathrm{~g}$ for $5 \mathrm{~min}$ to remove cell debris and filtered with a $0.45 \mu \mathrm{m}$ polyethersulfone membrane filter. Ultracentrifugation to concentrate the virus was performed for 2 hours at $12,000 \mathrm{~g}$ and $4^{\circ} \mathrm{C}$ using Beckman SW28 rotor. Lentivirus titers were measured by p24 specific enzyme-linked immunosorbent assay.

\section{Establishment of Triple Mutant Stable Cell Lines}

579 HEK293 cells stably expressing exogenous MTOR (wildtype or P2229R mutant) were transduced with NFkB2 580 (wild type or P882Q mutant) expressing lentiviruses. Infections were performed in the presence of $8 \mu \mathrm{g}$ $581 / \mathrm{mL}$ of polybrene under centrifugation $\left(500 \mathrm{~g}, 37^{\circ} \mathrm{C}\right)$ for 2 hours. MTOR-NFkB2 transduced cells (expressing 
MTOR-NFkB2 (wildtype or mutant) were infected with TLR2 (wildtype or W558L mutant) expressing

lentiviruses as described above and selected using puromycin $(3 \mu \mathrm{g} / \mathrm{mL})$.

\section{Western Blot Analysis}

After removing serum containing medium, HEK293 cells were washed twice with ice-cold PBS followed by

serum starvation for 12 hours. Cells were then harvested and further lysed in ice-cold RIPA buffer with $1 X$

protease and phosphatase inhibitor cocktail (Thermo Fisher Scientific). To remove cell debris,

centrifugation was carried out for $10 \mathrm{~min}$ at $4{ }^{\circ} \mathrm{C}, 12,000 \mathrm{~g}$. Total protein concentration was measured with the Qubit protein assay (Thermo Fisher Scientific) and $5 \mu \mathrm{g}$ of protein per sample was prepared in Laemmil buffer (Bio-Rad Laboratories) to load on a SDS-PAGE gel (Bio-Rad Laboratories). After running the sample in the SDS-PAGE gel, the proteins were transferred into a nitrocellulose membrane (Merk Millipore) followed

antibodies (1:1000 dilution) were incubated overnight at $4^{\circ} \mathrm{C}$ in PBS with $0.1 \%$ Tween 20 containing 5\% milk, and subsequently secondary antibodies (1:15,000 dilution) in PBS with $0.1 \%$ Tween 20 containing 5\% milk

\section{Drug Sensitivity and Resistance Testing (DSRT)}

601 Ex-vivo DSRT was performed on freshly isolated CD4+ T cells with a total of 527 drugs in 5 concentrations 602 covering a 10,000-fold concentration range including conventional chemotherapeutics and a broad range 603 of targeted oncology compounds.(47) To dissolve the drug compounds, $5 \mu$ l of medium was dispensed into 604 each well of 384 well plates including five different concentrations of each drug. $20 \mu \mathrm{l}$ of cell suspension 605 (CD4+ T cells from healthy control, donor and index patient: 2,000 cells per well) was transferred to every 
was evaluated by CellTiter-Glo Assay solution (Promega). The drug sensitivity score (DSS) was calculated to evaluate quantitative drug profiles based on the measured dose-response curve.(61)

\section{Reverse Transcriptase-quantitative Polymerase Chain Reaction (RT-qPCR)}

613 Total RNA was extracted using the RNeasy Mini kit (Qiagen) followed by cDNA synthesis using QuantiNova

614 Reverse Transcription kit (Qiagen) according to the manufacturer's protocol. The cDNA was applied in SYBR 615 Green RT-PCR master mix (Applied Biosystems) and oligonucleotide primers (Supplementary Table S6). All

616 RT-qPCR reactions were performed in 384-microwell plates (Applied Biosystem) using a QuantStudio 6 Flex

617 Real-Time PCR system (Applied Biosystems). The relative quantitation of gene expression was analyzed

618 using comparative cycle threshold $(\triangle \Delta C T)$ method, and beta actin (ACTB) was used as an endogenous

619 control to normalize gene expression level.

620

\section{Data availability}

Patient whole-exome and RNA-sequencing raw data related to table 1 and figure 4 are available from the corresponding author upon request owing to regulations pertaining to the authors ethics permit and deposition of these data in public repositories.

Statistical Analysis

627 Unpaired two-sided t-tests were performed using GraphPad Prism 6 for Mac OS X, version 6.0. In all 628 analyses, $P$-value $<0.05$ was considered as statistically significant. 
List of Supplementary Materials:

632 Supplementary results: Clinical Characteristics of the Index cGvHD Patient

633 Supplementary Figure S1. Medical history of the index patient

634 Supplementary Figure S2. Flow cytometry analysis of the index patient

635 Supplementary Figure S3. Variant allele frequencies of TLR2 mutations in CGVHD patients' and healthy

636 controls' $\mathrm{CD} 4+$ and $\mathrm{CD} 8+\mathrm{T}$ cells

637 Supplementary Figure S4. Functional analysis of wild type and TLR2 mutants in HEK293 cell line

638

639 Supplementary Figure S5. Gene expression fold change within CD4+ cell clusters between 2015 and 2017.

640

641 Supplementary Table S1. Summary of study cohorts

642 Supplementary Table S2. Gene list in the immunogene panel sequencing.

643 Supplementary Table S3. Primer sets of mTOR, NFkB2 and TLR2 amplicon sequencing

644 Supplementary Table S4. Primer set for mTOR, NFkB2 and TLR2 capillary sequencing

645 Supplementary Table S5. List of mTOR, NFkB2 and TLR2 mutagenesis primers

646 Supplementary Table S6. Primer list of RT-qPCR

647 Supplementary Table S7. Immunogene panel sequencing result for the index patient

648 Supplementary Table S8. Somatic NFKB2, TLR2 and MTOR mutations in different cellular fractions from the

649 index patient validated by amplicon sequencing

650 Supplementary Table S9. Somatic TLR2 mutation in CD4+ T-cells validated by amplicon sequencing

651

652 Supplementary Table S10. Somatic TLR2 mutation in CD8+ T-cells validated by amplicon sequencing 653

654 Supplementary Table S11. Gene set enrichment analysis (GSEA) 


\section{Acknowledgements}

656 Funding: This work was supported by the European Research Council (M-IMM project), Academy of

657 Finland, Finnish special governmental subsidy for health sciences, research and training, Sigrid Juselius

658 Foundation, Instrumentarium Science foundation, Helsinki Institute of Life Sciences Fellow funding, and

659 Finnish Cancer Institute. TL was supported by the Academy of Finland (Decisions 311081 and 314557). This

660 study was supported by Finnish Functional Genomics Centre, University of Turku, Åbo Akademi University

661 and Biocenter Finland.

662 Author Contributions: G.P., D.K., S.L., R.K., M.K. and S.M. designed the study and experiments. A.M.H.,

663 C.M-C., L.C., V.G.G.S., T.H.C-L., A.K., A.J., U.S., and M.I-R. contributed and prepared biological samples and

664 clinical data. G.P., D.K., S.L., R.K., O.B., J.H., and T.L. performed experiments and analyzed the data. P.E. and

665 S.H. designed, supervised and performed sequencing assays. S.E. performed bioinformatic analyses. S.M.

666 conceived and designed the study, directed and supervised research. G.P, M.K., and S.M. wrote the

667 manuscript. All authors read and approved the final manuscript.

668 Competing Interests statement: S.M. has received honoraria and research funding from Novartis, Pfizer

669 and Bristol-Myers Squibb (not related to this study). The remaining authors declare no competing interests.

670 Data and materials availability: Patient whole-exome sequencing and RNA-seq data are available from the

671 corresponding author upon suitable request. All other data associated with this study are available in the

672 main text or the supplementary materials.

673 


\section{REFERENCES}

675

1. J. R. Passweg, H. Baldomero, P. Bader, C. Bonini, S. Cesaro, P. Dreger, R. F. Duarte, C. Dufour, J. Kuball, D. Farge-Bancel, A. Gennery, N. Kroger, F. Lanza, A. Nagler, A. Sureda, M. Mohty, Hematopoietic stem cell transplantation in Europe 2014: more than 40000 transplants annually. Bone Marrow Transplant 51, 786-792 (2016).

2. B. R. Blazar, W. J. Murphy, M. Abedi, Advances in graft-versus-host disease biology and therapy. Nat Rev Immunol 12, 443-458 (2012).

3. K. R. Cooke, L. Luznik, S. Sarantopoulos, F. T. Hakim, M. Jagasia, D. H. Fowler, M. R. M. van den Brink, J. A. Hansen, R. Parkman, D. B. Miklos, P. J. Martin, S. Paczesny, G. Vogelsang, S. Pavletic, J. Ritz, K. R. Schultz, B. R. Blazar, The Biology of Chronic Graft-versus-Host Disease: A Task Force Report from the National Institutes of Health Consensus Development Project on Criteria for Clinical Trials in Chronic Graft-versus-Host Disease. Biol Blood Marrow Transplant 23, 211-234 (2017).

4. R. S. Goldszmid, G. Trinchieri, The price of immunity. Nat Immunol 13, $932-938$ (2012).

5. F. Pandolfi, R. Cianci, F. Casciano, D. Pagliari, T. De Pasquale, R. Landolfi, G. Di Sante, J. T. Kurnick, F. Ria, Skewed T-cell receptor repertoire: more than a marker of malignancy, a tool to dissect the immunopathology of inflammatory diseases. J Biol Regul Homeost Agents 25, 153-161 (2011).

6. P. Savola, T. Kelkka, H. L. Rajala, A. Kuuliala, K. Kuuliala, S. Eldfors, P. Ellonen, S. Lagstrom, M. Lepisto, T. Hannunen, E. I. Andersson, R. K. Khajuria, T. Jaatinen, R. Koivuniemi, H. Repo, J. Saarela, K. Porkka, M. Leirisalo-Repo, S. Mustjoki, Somatic mutations in clonally expanded cytotoxic T lymphocytes in patients with newly diagnosed rheumatoid arthritis. Nat Commun $\mathbf{8}$, 15869 (2017).

7. Y. J. Zhang, Y. Duan, X. F. Zheng, Targeting the mTOR kinase domain: the second generation of mTOR inhibitors. Drug Discov Today 16, 325-331 (2011).

8. S. C. Sun, The non-canonical NF-kappaB pathway in immunity and inflammation. Nat Rev Immunol 17, 545-558 (2017).

9. U. Platzbecker, J. Stoehlmacher, C. Pabst, E. Goekkurt, U. Oelschlagel, H. Schirutschke, K. Holig, C. Theuser, U. Mogck, G. Ehninger, M. Bornhauser, Induction of Toll-like receptor 2 and 4 expression on CD4+ and CD8+ T cells in G-CSF-mobilized unrelated peripheral blood stem cell grafts during leukapheresis: impact on patient outcome. Leukemia 22, 1438-1440 (2008).

10. H. L. Rajala, T. Olson, M. J. Clemente, S. Lagstrom, P. Ellonen, T. Lundan, D. E. Hamm, S. A. Zaman, J. M. Lopez Marti, E. I. Andersson, A. Jerez, K. Porkka, J. P. Maciejewski, T. P. Loughran, S. Mustjoki, The analysis of clonal diversity and therapy responses using STAT3 mutations as a molecular marker in large granular lymphocytic leukemia. Haematologica 100, $91-99$ (2015).

11. H. B. Jefferies, S. Fumagalli, P. B. Dennis, C. Reinhard, R. B. Pearson, G. Thomas, Rapamycin suppresses 5'TOP mRNA translation through inhibition of p70s6k. EMBO J 16, 3693-3704 (1997).

12. D. D. Sarbassov, D. A. Guertin, S. M. Ali, D. M. Sabatini, Phosphorylation and regulation of Akt/PKB by the rictor-mTOR complex. Science 307, 1098-1101 (2005).

13. B. C. Grabiner, V. Nardi, K. Birsoy, R. Possemato, K. Shen, S. Sinha, A. Jordan, A. H. Beck, D. M. Sabatini, A diverse array of cancer-associated MTOR mutations are hyperactivating and can predict rapamycin sensitivity. Cancer Discov 4, 554-563 (2014).

14. S. Gerondakis, T. S. Fulford, N. L. Messina, R. J. Grumont, NF-kappaB control of T cell development. Nat Immunol 15, 15-25 (2014).

15. B. Hwang, J. H. Lee, D. Bang, Single-cell RNA sequencing technologies and bioinformatics pipelines. Exp Mol Med 50, 96 (2018).

16. J. Glamann, A. J. Hansen, Dynamic detection of natural killer cell-mediated cytotoxicity and cell adhesion by electrical impedance measurements. Assay Drug Dev Technol 4, 555-563 (2006). 
17. A. Kreutzman, B. Colom-Fernandez, A. M. Jimenez, M. Ilander, C. Cuesta-Mateos, Y. Perez-Garcia, C. D. Arevalo, O. Bruck, H. Hakanen, J. Saarela, A. Ortega-Carrion, A. de Rosendo, A. JuanesGarcia, J. L. Steegmann, S. Mustjoki, M. Vicente-Manzanares, C. Munoz-Calleja, Dasatinib Reversibly Disrupts Endothelial Vascular Integrity by Increasing Non-Muscle Myosin II Contractility in a ROCK-Dependent Manner. Clin Cancer Res 23, 6697-6707 (2017).

18. T. Pemovska, M. Kontro, B. Yadav, H. Edgren, S. Eldfors, A. Szwajda, H. Almusa, M. M. Bespalov, P. Ellonen, E. Elonen, B. T. Gjertsen, R. Karjalainen, E. Kulesskiy, S. Lagstrom, A. Lehto, M. Lepisto, T. Lundan, M. M. Majumder, J. M. Marti, P. Mattila, A. Murumagi, S. Mustjoki, A. Palva, A. Parsons, T. Pirttinen, M. E. Ramet, M. Suvela, L. Turunen, I. Vastrik, M. Wolf, J. Knowles, T. Aittokallio, C. A. Heckman, K. Porkka, O. Kallioniemi, K. Wennerberg, Individualized systems medicine strategy to tailor treatments for patients with chemorefractory acute myeloid leukemia. Cancer Discov 3, 1416-1429 (2013).

19. I. Martincorena, P. J. Campbell, Somatic mutation in cancer and normal cells. Science 349, 14831489 (2015).

20. M. Xie, C. Lu, J. Wang, M. D. McLellan, K. J. Johnson, M. C. Wendl, J. F. McMichael, H. K. Schmidt, V. Yellapantula, C. A. Miller, B. A. Ozenberger, J. S. Welch, D. C. Link, M. J. Walter, E. R. Mardis, J. F. Dipersio, F. Chen, R. K. Wilson, T. J. Ley, L. Ding, Age-related mutations associated with clonal hematopoietic expansion and malignancies. Nat Med 20, 1472-1478 (2014).

21. E. Holzelova, C. Vonarbourg, M. C. Stolzenberg, P. D. Arkwright, F. Selz, A. M. Prieur, S. Blanche, J. Bartunkova, E. Vilmer, A. Fischer, F. Le Deist, F. Rieux-Laucat, Autoimmune lymphoproliferative syndrome with somatic Fas mutations. N Engl J Med 351, 1409-1418 (2004).

22. K. A. Ross, Coherent somatic mutation in autoimmune disease. PLoS One 9, e101093 (2014).

23. S. I. Nikolaev, S. Vetiska, X. Bonilla, E. Boudreau, S. Jauhiainen, B. Rezai Jahromi, N. Khyzha, P. V. DiStefano, S. Suutarinen, T. R. Kiehl, V. Mendes Pereira, A. M. Herman, T. Krings, H. AndradeBarazarte, T. Tung, T. Valiante, G. Zadeh, M. Tymianski, T. Rauramaa, S. Yla-Herttuala, J. D. Wythe, S. E. Antonarakis, J. Frosen, J. E. Fish, I. Radovanovic, Somatic Activating KRAS Mutations in Arteriovenous Malformations of the Brain. N Engl J Med 378, 250-261 (2018).

24. H. L. Koskela, S. Eldfors, P. Ellonen, A. J. van Adrichem, H. Kuusanmaki, E. I. Andersson, S. Lagstrom, M. J. Clemente, T. Olson, S. E. Jalkanen, M. M. Majumder, H. Almusa, H. Edgren, M. Lepisto, P. Mattila, K. Guinta, P. Koistinen, T. Kuittinen, K. Penttinen, A. Parsons, J. Knowles, J. Saarela, K. Wennerberg, O. Kallioniemi, K. Porkka, T. P. Loughran, Jr., C. A. Heckman, J. P. Maciejewski, S. Mustjoki, Somatic STAT3 mutations in large granular lymphocytic leukemia. $N$ Engl J Med 366, 1905-1913 (2012).

25. J. A. Fraietta, C. L. Nobles, M. A. Sammons, S. Lundh, S. A. Carty, T. J. Reich, A. P. Cogdill, J. J. D. Morrissette, J. E. DeNizio, S. Reddy, Y. Hwang, M. Gohil, I. Kulikovskaya, F. Nazimuddin, M. Gupta, F. Chen, J. K. Everett, K. A. Alexander, E. Lin-Shiao, M. H. Gee, X. Liu, R. M. Young, D. Ambrose, Y. Wang, J. Xu, M. S. Jordan, K. T. Marcucci, B. L. Levine, K. C. Garcia, Y. Zhao, M. Kalos, D. L. Porter, R. M. Kohli, S. F. Lacey, S. L. Berger, F. D. Bushman, C. H. June, J. J. Melenhorst, Disruption of TET2 promotes the therapeutic efficacy of CD19-targeted T cells. Nature 558, 307-312 (2018).

26. F. Janku, T. A. Yap, F. Meric-Bernstam, Targeting the PI3K pathway in cancer: are we making headway? Nat Rev Clin Oncol 15, 273-291 (2018).

27. B. Blanco, M. C. Herrero-Sanchez, C. Rodriguez-Serrano, M. Sanchez-Barba, M. C. Del Canizo, Profound blockade of T cell activation requires concomitant inhibition of different class I PI3K isoforms. Immunol Res 62, 175-188 (2015).

28. A. Abouelnasr, J. Roy, S. Cohen, T. Kiss, S. Lachance, Defining the role of sirolimus in the management of graft-versus-host disease: from prophylaxis to treatment. Biol Blood Marrow Transplant 19, 12-21 (2013).

29. J. A. DiDonato, F. Mercurio, M. Karin, NF-kappaB and the link between inflammation and cancer. Immunol Rev 246, 379-400 (2012). 
30. Q. Zhang, M. J. Lenardo, D. Baltimore, 30 Years of NF-kappaB: A Blossoming of Relevance to Human Pathobiology. Cell 168, 37-57 (2017).

31. F. Christian, E. L. Smith, R. J. Carmody, The Regulation of NF-kappaB Subunits by Phosphorylation. Cells 5, (2016).

32. T. H. Flo, O. Halaas, S. Torp, L. Ryan, E. Lien, B. Dybdahl, A. Sundan, T. Espevik, Differential expression of Toll-like receptor 2 in human cells. J Leukoc Biol 69, 474-481 (2001).

33. M. Komai-Koma, L. Jones, G. S. Ogg, D. Xu, F. Y. Liew, TLR2 is expressed on activated T cells as a costimulatory receptor. Proc Natl Acad Sci U S A 101, 3029-3034 (2004).

34. S. P. Cullen, M. Brunet, S. J. Martin, Granzymes in cancer and immunity. Cell Death Differ 17, 616$623(2010)$.

35. D. M. Brown, A. T. Lampe, A. M. Workman, The Differentiation and Protective Function of Cytolytic CD4 T Cells in Influenza Infection. Front Immunol 7, 93 (2016).

36. H. Cheroutre, M. M. Husain, CD4 CTL: living up to the challenge. Semin Immunol 25, 273-281 (2013).

37. V. S. Patil, A. Madrigal, B. J. Schmiedel, J. Clarke, P. O'Rourke, A. D. de Silva, E. Harris, B. Peters, G. Seumois, D. Weiskopf, A. Sette, P. Vijayanand, Precursors of human CD4(+) cytotoxic T lymphocytes identified by single-cell transcriptome analysis. Sci Immunol 3, (2018).

38. W. Wei, Y. Jiao, A. Postlethwaite, J. M. Stuart, Y. Wang, D. Sun, W. Gu, Dual-specificity phosphatases 2: surprising positive effect at the molecular level and a potential biomarker of diseases. Genes Immun 14, 1-6 (2013).

39. D. Lu, L. Liu, X. Ji, Y. Gao, X. Chen, Y. Liu, Y. Liu, X. Zhao, Y. Li, Y. Li, Y. Jin, Y. Zhang, M. A. McNutt, Y. Yin, The phosphatase DUSP2 controls the activity of the transcription activator STAT3 and regulates TH17 differentiation. Nat Immunol 16, 1263-1273 (2015).

40. R. Lang, M. Hammer, J. Mages, DUSP meet immunology: dual specificity MAPK phosphatases in control of the inflammatory response. J Immunol 177, 7497-7504 (2006).

41. A. M. Fischer, C. D. Katayama, G. Pages, J. Pouyssegur, S. M. Hedrick, The role of erk1 and erk2 in multiple stages of T cell development. Immunity 23, 431-443 (2005).

42. X. He, R. L. Smeets, H. J. Koenen, P. M. Vink, J. Wagenaars, A. M. Boots, I. Joosten, Mycophenolic acid-mediated suppression of human CD4+ T cells: more than mere guanine nucleotide deprivation. Am J Transplant 11, 439-449 (2011).

43. D. A. Guertin, D. M. Sabatini, Defining the role of mTOR in cancer. Cancer Cell 12, 9-22 (2007).

44. C. K. Tsang, H. Qi, L. F. Liu, X. F. Zheng, Targeting mammalian target of rapamycin (mTOR) for health and diseases. Drug Discov Today 12, 112-124 (2007).

45. M. A. Bjornsti, P. J. Houghton, The TOR pathway: a target for cancer therapy. Nat Rev Cancer 4, 335-348 (2004).

46. A. Perl, mTOR activation is a biomarker and a central pathway to autoimmune disorders, cancer, obesity, and aging. Ann N Y Acad Sci 1346, 33-44 (2015).

47. X. F. Zheng, D. Florentino, J. Chen, G. R. Crabtree, S. L. Schreiber, TOR kinase domains are required for two distinct functions, only one of which is inhibited by rapamycin. Cell $\mathbf{8 2}, 121-130$ (1995).

48. M. E. Feldman, B. Apsel, A. Uotila, R. Loewith, Z. A. Knight, D. Ruggero, K. M. Shokat, Activesite inhibitors of mTOR target rapamycin-resistant outputs of mTORC1 and mTORC2. PLoS Biol 7, e38 (2009).

49. C. C. Thoreen, S. A. Kang, J. W. Chang, Q. Liu, J. Zhang, Y. Gao, L. J. Reichling, T. Sim, D. M. Sabatini, N. S. Gray, An ATP-competitive mammalian target of rapamycin inhibitor reveals rapamycin-resistant functions of mTORC1. J Biol Chem 284, 8023-8032 (2009).

50. M. Taipale, D. F. Jarosz, S. Lindquist, HSP90 at the hub of protein homeostasis: emerging mechanistic insights. Nat Rev Mol Cell Biol 11, 515-528 (2010).

51. Y. Fukuyo, C. R. Hunt, N. Horikoshi, Geldanamycin and its anti-cancer activities. Cancer Lett 290, 24-35 (2010). 
52. P. C. Echeverria, A. Bernthaler, P. Dupuis, B. Mayer, D. Picard, An interaction network predicted from public data as a discovery tool: application to the Hsp90 molecular chaperone machine. PLoS One 6, e26044 (2011).

53. G. M. Delgoffe, T. P. Kole, R. J. Cotter, J. D. Powell, Enhanced interaction between Hsp90 and raptor regulates mTOR signaling upon T cell activation. Mol Immunol 46, 2694-2698 (2009).

54. S. Sato, N. Fujita, T. Tsuruo, Modulation of Akt kinase activity by binding to Hsp90. Proc Natl Acad Sci U S A 97, 10832-10837 (2000).

55. H. Bekki, K. Kohashi, A. Maekawa, Y. Yamada, H. Yamamoto, K. Harimaya, M. Hakozaki, K. Nabeshima, Y. Iwamoto, Y. Oda, Elevated expression of HSP90 and the antitumor effect of an HSP90 inhibitor via inactivation of the Akt/mTOR pathway in undifferentiated pleomorphic sarcoma. BMC Cancer 15, 804 (2015).

56. L. Giulino-Roth, H. J. van Besien, T. Dalton, J. E. Totonchy, A. Rodina, T. Taldone, A. Bolaender, H. Erdjument-Bromage, J. Sadek, A. Chadburn, M. J. Barth, F. S. Dela Cruz, A. Rainey, A. L. Kung, G. Chiosis, E. Cesarman, Inhibition of Hsp90 Suppresses PI3K/AKT/mTOR Signaling and Has Antitumor Activity in Burkitt Lymphoma. Mol Cancer Ther 16, 1779-1790 (2017).

57. Q. Huang, S. He, Y. Tian, Y. Gu, P. Chen, C. Li, J. Huang, Y. Liu, H. Yu, M. Jin, S. Hu, Q. Tong, A. Ma, J. Jin, E. Hexner, H. Fung, R. Reshef, Y. Zhang, Y. Zhang, Hsp90 inhibition destabilizes Ezh2 protein in alloreactive T cells and reduces graft-versus-host disease in mice. Blood 129, 2737-2748 (2017).

58. C. S. Carlson, R. O. Emerson, A. M. Sherwood, C. Desmarais, M. W. Chung, J. M. Parsons, M. S. Steen, M. A. LaMadrid-Herrmannsfeldt, D. W. Williamson, R. J. Livingston, D. Wu, B. L. Wood, M. J. Rieder, H. Robins, Using synthetic templates to design an unbiased multiplex PCR assay. Nat Commun 4, 2680 (2013).

59. C. Soneson, M. D. Robinson, Bias, robustness and scalability in single-cell differential expression analysis. Nat Methods 15, 255-261 (2018).

60. M. Vangipuram, D. Ting, S. Kim, R. Diaz, B. Schule, Skin punch biopsy explant culture for derivation of primary human fibroblasts. $J$ Vis Exp, e3779 (2013).

61. B. Yadav, T. Pemovska, A. Szwajda, E. Kulesskiy, M. Kontro, R. Karjalainen, M. M. Majumder, D. Malani, A. Murumagi, J. Knowles, K. Porkka, C. Heckman, O. Kallioniemi, K. Wennerberg, T. Aittokallio, Quantitative scoring of differential drug sensitivity for individually optimized anticancer therapies. Sci Rep 4, 5193 (2014). 
Table 1. Somatic mutations discovered in CD4+ T cells in the index patient, detected from 2013 sample

\begin{tabular}{|c|c|c|c|c|c|c|c|c|c|c|c|c|c|c|c|}
\hline $\mathrm{Chr}$ & position & ref & var & Gene & $\begin{array}{c}\text { Mutation } \\
\text { type }\end{array}$ & $\begin{array}{l}\text { Codon } \\
\text { change }\end{array}$ & Exon & $\begin{array}{l}\text { Amino } \\
\text { acid } \\
\text { change }\end{array}$ & $\begin{array}{l}\text { Normal_-1 } \\
\text { Ref reads }\end{array}$ & $\begin{array}{l}\text { Normal-2 } \\
\text { Alt reads }\end{array}$ & $\begin{array}{l}\text { Normal-var_- } \\
\text { Freq (\%) }\end{array}$ & $\begin{array}{c}\text { Tumor_. }^{\text {The reads }}{ }^{3} \\
\text { Ref }\end{array}$ & $\begin{array}{c}\text { Tumor__ } \\
\text { Alt reads }\end{array}$ & $\begin{array}{c}\text { Tumor_var_- } \\
\text { Freq (\%) }\end{array}$ & $\begin{array}{l}\text { Somatic } \\
\text { p-value }\end{array}$ \\
\hline 1 & 11182160 & $G$ & c & MTOR & MISSENSE & $\mathrm{cct} / \mathrm{cGt}$ & 48 & P2229R & 179 & 8 & 4.28 & 157 & 24 & 13.26 & 0.0017815 \\
\hline 4 & 154625732 & G & $\mathrm{T}$ & TLR2 & MISSENSE & $\mathrm{tGg} / \mathrm{tg}$ & 1 & W558L & 116 & 2 & 1.69 & 104 & 10 & 8.77 & 0.014504 \\
\hline 4 & 144801662 & c & G & GYPE & MISSENSE & $\mathrm{gGa} / \mathrm{gCa}$ & 2 & G13A & 150 & 68 & 31.19 & 147 & 99 & 40.24 & 0.026604 \\
\hline 19 & 50017643 & G & $T$ & FCGRT & MISSENSE & $\mathrm{caG} / \mathrm{cat}$ & 3 & $\mathrm{Q} 167 \mathrm{H}$ & 28 & 0 & 0 & 14 & 2 & 12.5 & 0.12685 \\
\hline 16 & 31388150 & A & $G$ & ITGAX & MISSENSE & Aca/Gca & 21 & T847A & 57 & 0 & 0 & 35 & 2 & 5.41 & 0.15237 \\
\hline 11 & 2415324 & G & $\mathrm{T}$ & CD81 & SPLICING & & 4 & & 58 & 0 & 0 & 36 & 2 & 5.26 & 0.15417 \\
\hline 22 & 37326772 & c & A & CSF $2 R B$ & MISSENSE & $\mathrm{cac} / \mathrm{caA}$ & 8 & H310Q & 54 & 0 & 0 & 35 & 2 & 5.41 & 0.16264 \\
\hline 3 & 49936028 & A & c & MSTIR & MISSENSE & $\mathrm{Tgt} / \mathrm{Ggt}$ & 4 & C548G & 45 & 0 & 0 & 38 & 2 & 5 & 0.21849 \\
\hline 10 & 104162075 & c & A & NFKB2 & MISSENSE & cCa/cAa & 23 & P882Q & 14 & 0 & 0 & 26 & 3 & 10.34 & 0.29609 \\
\hline 10 & 18112382 & G & $\mathrm{T}$ & MRC1 & MISSENSE & Ggt/Tgt & 2 & G134C & 29 & 0 & 0 & 35 & 2 & 5.41 & 0.31049 \\
\hline 17 & 80274159 & $\mathrm{G}$ & GT & $C D 7$ & $\begin{array}{l}\text { FRAME } \\
\text { SHIIFT }\end{array}$ & $\mathrm{gca} / \mathrm{gAca}$ & 3 & A175D? & 8 & 0 & 0 & 16 & 3 & 15.79 & 0.33128 \\
\hline 17 & 80274183 & G & c & $C D 7$ & MISSENSE & $\mathrm{gCc} / \mathrm{GGc}$ & 3 & A167G & 13 & 0 & 0 & 19 & 2 & 9.52 & 0.37433 \\
\hline 12 & 109017698 & G & A & SELPLG & MISSENSE & $\mathrm{acg} / \mathrm{TTg}$ & 2 & $\mathrm{~T} 145 \mathrm{M}$ & 46 & 2 & 4.17 & 35 & 3 & 7.89 & 0.38939 \\
\hline 17 & 80274161 & TG & ${ }^{\top}$ & $C D 7$ & $\begin{array}{l}\text { FRAME } \\
\text { SHIIFT }\end{array}$ & - & 3 & & 7 & 0 & 0 & 11 & 2 & 15.38 & 0.41053 \\
\hline
\end{tabular}

858 Immunogene panel sequencing was performed on both CD4+ and CD8+ T cells from the index cGvHD

859 patient. The table shows discovered somatic mutations in the expanded CD4+ T cells.

861 Abbreviations: Chr, chromosome; ref, reference base; var, variant base; freq, frequency

$862{ }^{1}$ Sequencing reads supporting reference allele in normal sample, ${ }^{2}$ Sequencing reads supporting variant

863 allele in normal sample, ${ }^{3}$ Sequencing reads supporting reference allele in tumor sample, ${ }^{4}$ Sequencing

864 reads supporting variant allele in tumor sample, ${ }^{*}$ Somatic $p$-value for somatic/loss of heterozygosity

865 events 
Table 2. Somatic MTOR and NFkB2 mutations validated by amplicon sequencing

\begin{tabular}{|c|c|c|c|c|c|c|c|c|c|c|c|c|c|}
\hline $\begin{array}{c}\text { Sample } \\
\text { year }\end{array}$ & Patient & DNAs & Gene & $\mathrm{Chr}$ & position & ref & var & $\begin{array}{c}\text { Amino acid } \\
\text { change }\end{array}$ & Call_Depth & Ref_Calls & Var_Calls & $\begin{array}{l}\text { VAF } \\
\text { (\%) }\end{array}$ & Freq_Ratio \\
\hline 2015 & $1-1$ & $\mathrm{CD} 4+\mathrm{T}$ & MTOR & 1 & 112182160 & G & c & P2229R & 1000004 & 808220 & 191784 & 19.2 & 1.00047 \\
\hline 2015 & $1-2$ & $\mathrm{CD} 8+\mathrm{T}$ & MTOR & 1 & 112182160 & G & c & P2229R & 1000003 & 942683 & 57320 & 5.7 & 0.9993 \\
\hline 2017 & $1-3$ & $\begin{array}{c}\text { CD4+ } \\
\text { Vb.20+ }\end{array}$ & MTOR & 1 & 112182160 & G & c & P2229R & 168592 & 93183 & 75409 & 44.7 & 0.99928 \\
\hline 2017 & $1-4$ & $\begin{array}{c}\text { CD4+CD8+ } \\
\text { Vb.20+ }\end{array}$ & MTOR & 1 & 112182160 & G & c & P2229R & 171728 & 110670 & 61058 & 35.5 & 0.99910 \\
\hline 2015 & $1-5$ & Skin-biopsy & MTOR & 1 & 112182160 & G & c & P2229R & 8085 & 7986 & 99 & 0.8 & 0.96305 \\
\hline 2016 & 2 & Whole blood & MTOR & 1 & 112182160 & G & c & P2229R & 2743 & 2715 & 28 & 1.0 & 1.01793 \\
\hline 2015 & 3 & Whole blood & MTOR & 1 & 112182160 & G & c & P2229R & 719 & 703 & 16 & 2.0 & 1.02671 \\
\hline 2015 & $1-1$ & $\mathrm{CD} 4+\mathrm{T}$ & NFkB2 & 10 & 104162075 & C & A & P882Q & 471375 & 414821 & 56554 & 12.0 & 1.0061 \\
\hline 2015 & $1-2$ & $\mathrm{CD} 8+\mathrm{T}$ & NFkB2 & 10 & 104162075 & C & A & P882Q & 586903 & 577283 & 9620 & 1.6 & 1.00124 \\
\hline 2017 & $1-3$ & $\begin{array}{c}\text { CD4+ } \\
\text { Vb.20+ }\end{array}$ & NFkB2 & 10 & 104162075 & C & A & P882Q & 26262 & 20668 & 5594 & 21.3 & 1.00395 \\
\hline 2017 & $1-4$ & $\begin{array}{c}\text { CD4+CD8+ } \\
\text { Vb.20+ }\end{array}$ & NFkB2 & 10 & 104162075 & C & A & P882Q & 48654 & 43822 & 4832 & 9.9 & 1.00282 \\
\hline
\end{tabular}

867

868 CD4+ and CD8+ T cells were sorted either with the magnetic beads (2015 sample) or flow based sorting

869 (2017 sample). In addition, $C D 4+V b 20+$ and $C D 4+C D 8+V b 20+$ fractions were sorted with flow cytometry

870 (2017 sample). MTOR and NFKB2 mutations were analysed from sorted fractions with deep amplicon

sequencing. Mutations were confined to CD4+ fractions. The low mutation VAFs in CD8+ fraction are due

to small CD4+ T cell contamination (CD4+CD8+ double positive cells) in the bead sorted fraction.

Abbreviations: Chr, chromosome; ref, reference base; var, variant base; Call_depth, total number of called 
bioRxiv preprint doi: https://doi.org/10.1101/747196; this version posted August 31,2019. The copyright holder for this preprint (which was not certified by peer review) is the author/funder. All rights reserved. No reuse allowed without permission.

Figure 1.

A

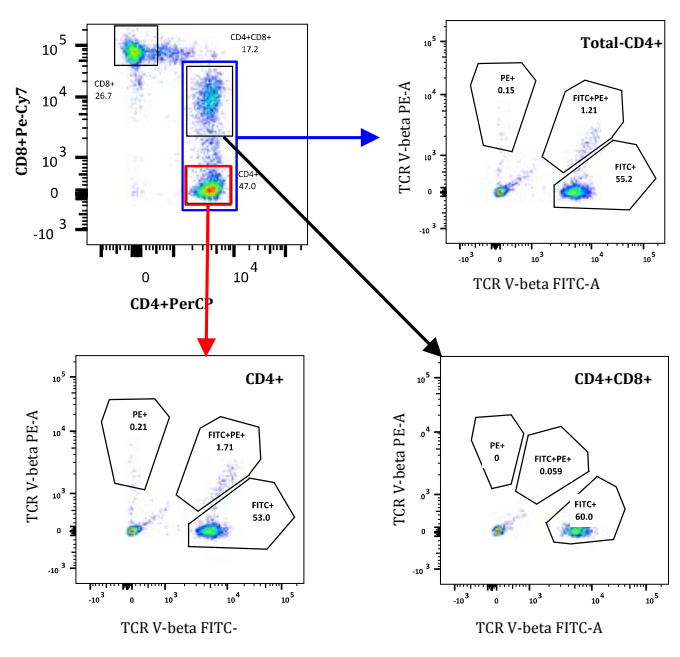

C

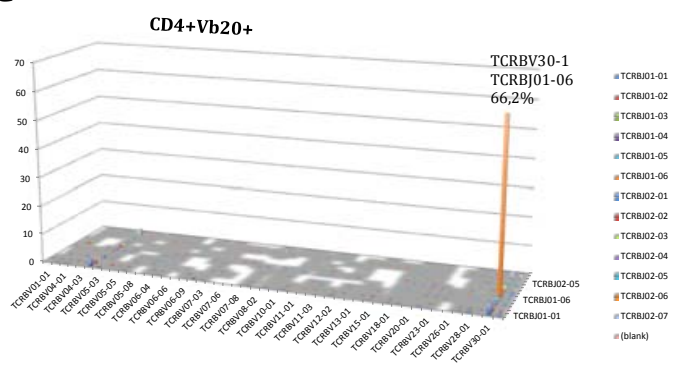

D
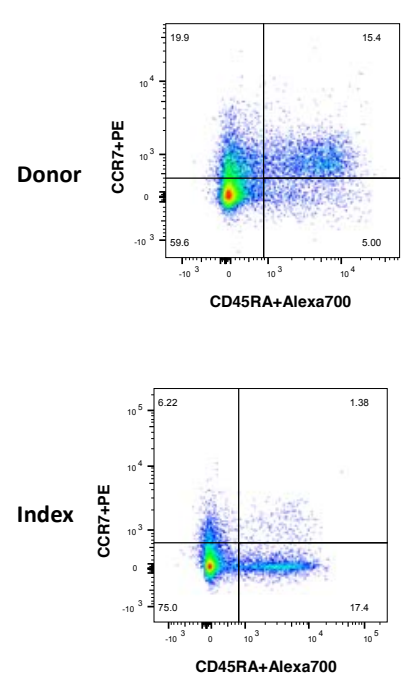

B
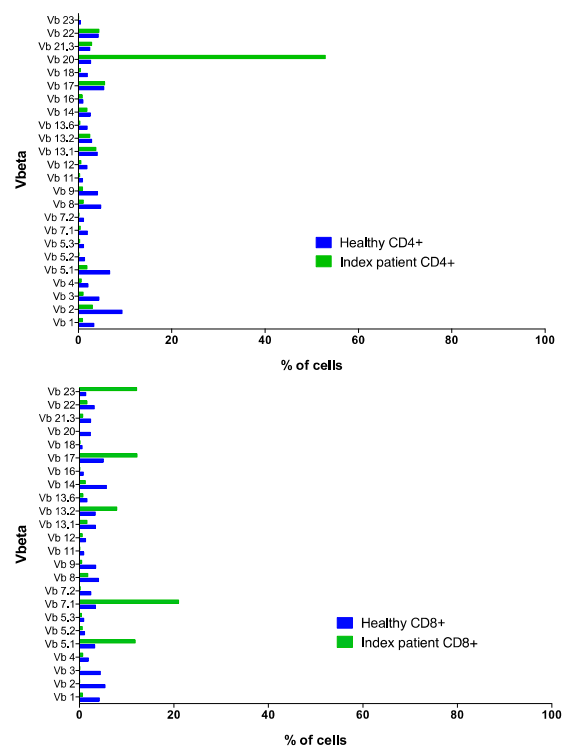

CD8+

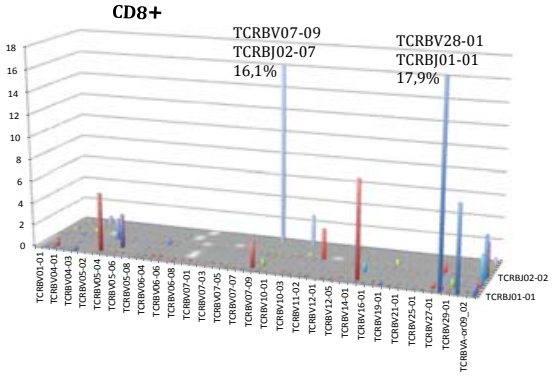

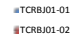


881 Figure 1. Flow cytometry and TCRB deep sequencing results from the index patient.

882 (A) and (B). TCR V $\beta$ repertoire of CD4+ and CD8+ T cells was analyzed in peripheral

883 blood from the Index patient with the IO Test Beta Mark TCR beta Repertoire Kit

884 (Beckman-Coulter Immunotech, USA). $53 \%$ of CD4+T cells and $60 \%$ of CD4+CD8+T

885 cells consisted of a single $\mathrm{V} \beta 20$ clone.

886 (C) T cell repertoire of FACS-sorted CD4+V $\beta 20+$ and CD8+ T cells analysed with TCR $\beta$

887 deep sequencing (Adaptive Biotech., USA). The TCRBV30-01 clone was detected in

888 the $C D 4+V \beta 20+$ fraction, but not in the $C D 8+$ fraction.

889 (D) Multicolor flow cytometry was applied to identify the immune phenotype of

890 donor and index patient's memory T cell subtypes. Central memory (CM), naïve,

891 effector memory (EM) and terminal effector memory (TEMRA) cells.

892

893 


\section{Figure 2.}

895 A

896

897

mTOR

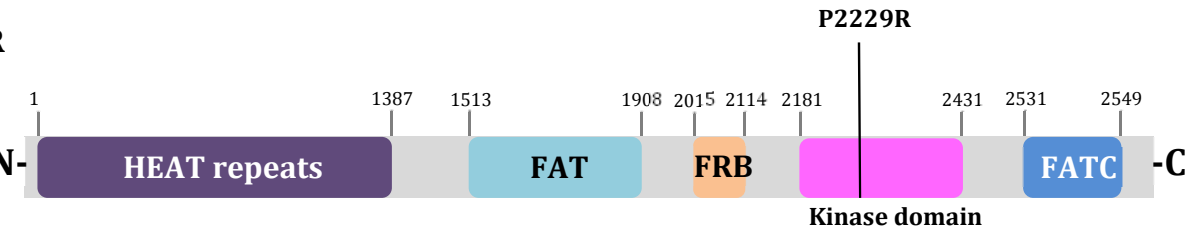

NFkB2

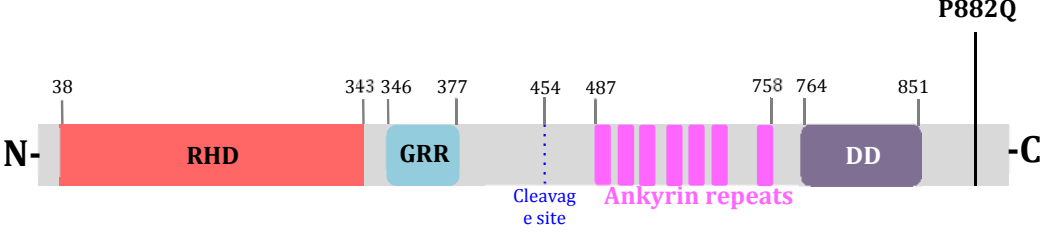

TLR2

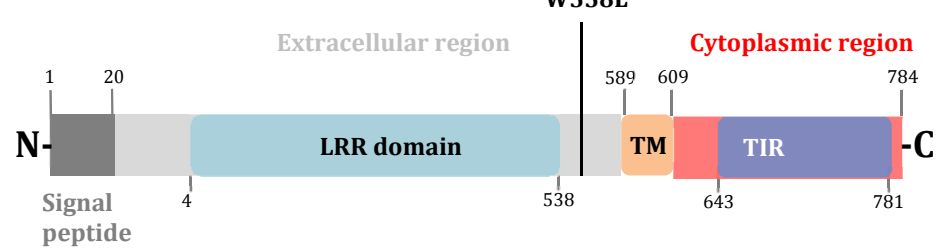

B

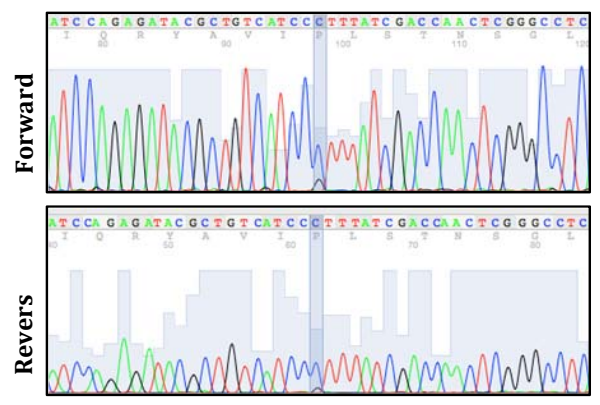

C

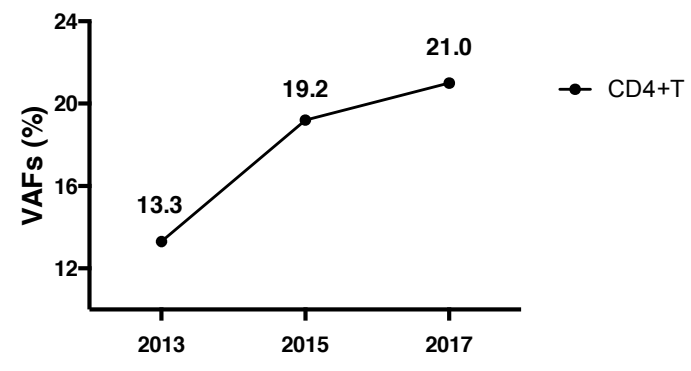

D

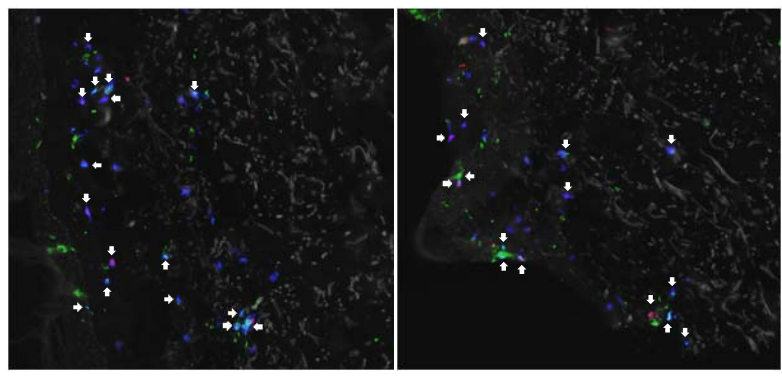


898 Figure 2. MTOR, NFkB2, and TLR2 mutations in index patient

899 (A) Locations of MTOR, TLR2, and NFkB2 somatic mutations. Linearized structure of

900 MTOR, NFkB2, and TLR2 presenting the location of somatic mutations. MTOR

$901 P 2229 R$ mutation is located in the kinase domain, NFkB2 P882Q in the C-terminus,

902 and TLR2 W558L between LRR (Leucine-rich repeats) domain and transmembrane

903 (TM) domain.

904 (B) A heterozygous MTOR mutation (G to C, P2229R) was detected in CD4+ T cells by

905 Sanger sequencing.

906 (C) Variant allele frequencies (VAFs) of MTOR mutation in the index patient's CD4+ T

907 cells over time as measured with amplicon sequencing.

908 (D) Immunofluorescence staining indicated CD3+CD4+ and CD3+CD8+T cell

909 infiltration in the skin. Paraffin embedded skin biopsy from index patient was

910 sectioned and stained with antibody specific human CD3 (cyan), CD4 (green), and

911 CD8 (red). White arrows indicate infiltrated CD3+CD4+ or CD3+CD8+T cells.

912 


\section{$913 \quad$ Figure 3.}

$914 \quad$ A

915

916

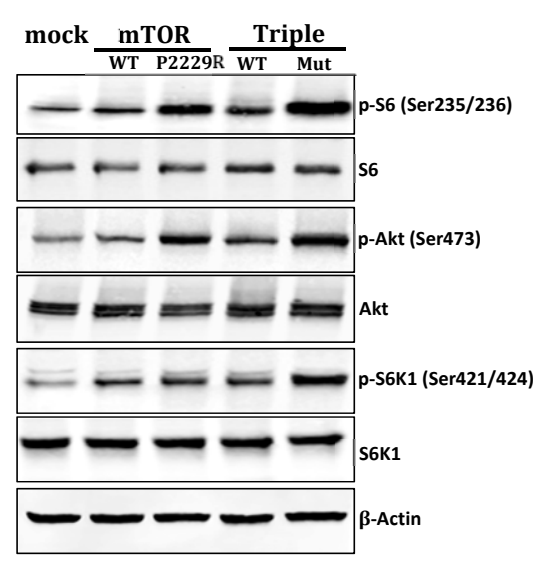

C

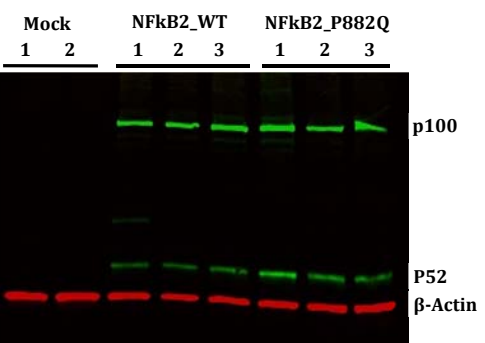

B
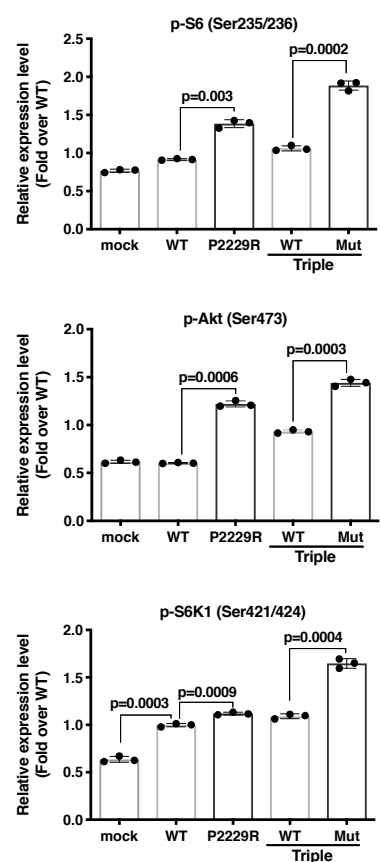

D

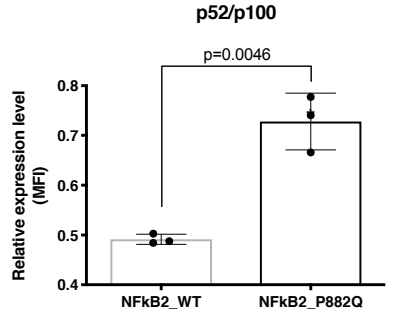


917 Figure 3. Functional analysis for wild type, MTOR, and NFkB2 mutants in HEK293

918 cells

919 (A) Stably expressed MTOR wildtype (WT), mutant (P2229R), Triple (MTOR, TLR2,

920 and NFKB2) wildtype (WT) and Triple mutant (MTOR P2229R, TLR2 W559L, and

921 NFKB2 P882Q) (Mut) in HEK293 cells were serum starved for 12 hours. Western blot

922 analysis was performed with the use of anti-pS6, anti-S6, anti-pAkt, anti-Akt, anti-

923 pS6K1, anti-S6K1, and anti- $\beta$-actin. Data is representative of three independent

924 experiments.

925 (B) Relative expression level was estimated by measuring each band intensity of

926 three independent experiments using ImageJ software (Rasband, W.S., ImageJ, U. S.

927 National Institutes of Health, Bethesda, Maryland, USA, https://imagej.nih.gov/ij/,

928 1997-2016). Error bar present Mean \pm SEM ( $n=3$ per group). $P$ values are derived

929 from unpaired t-test with Welch's correction (WT vs P2229R, WT vs Triple Mut, and

930 mock vs WT in p-S6K1).

931 (C) Immonoblot assay was performed to verify an alteration of P52 and P100

932 expression with NFkB2 WT and P882Q mutant. Data is representative of three

933 independent experiments.

934 (D) Stably expressing NFkB2 P882Q increased expression of P52. Mean fluorescence

935 intensity was measured by ImageJ software (NFkB2_WT: $n=3$, NFkB2_P882Q: $n=3$ ).

936 Error bar present Mean \pm SEM. P values are derived from unpaired t-test with

937 Welch's correction (NFkB2_WT vs NFkB2_P882Q).

938 
Figure 4.

940 A

941

942

943

944

945

946

947

948

949

950

951

952

953

954

955

956

957

958

959 F

960

961

962

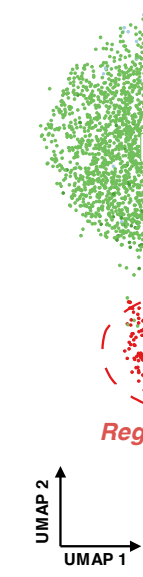

Naïve

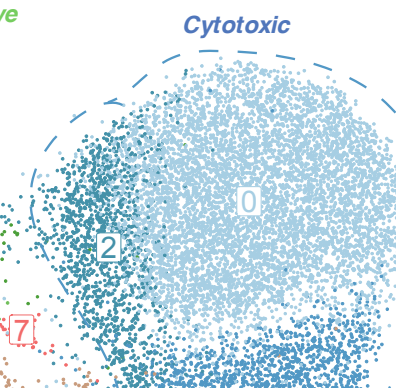

gulatory

$\overrightarrow{A P 1}$
B

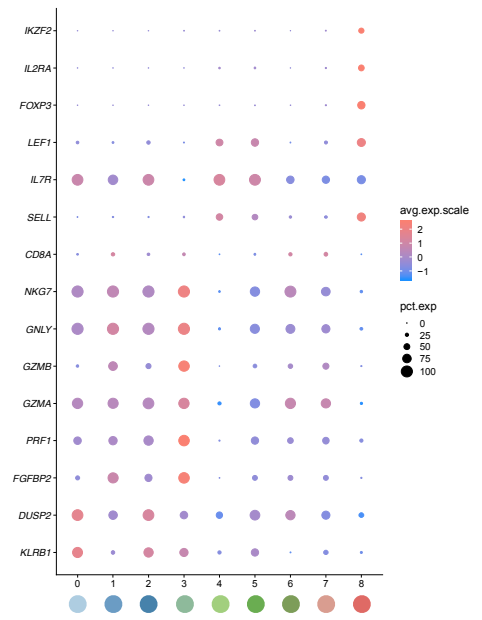

E 
963 Figure 4. Single-cell RNAseq analysis from the index patient.

964 (A) Two-dimension UMAP-projection of clustered CD4+ cells pooled from two time

965 points from peripheral blood. A total of 15,874 cells are annotated in 9 distinct

966 clusters.

967 B) Gene expression heatmap for the 9 distinct CD4+ clusters, where rows represent

968 canonical marker genes and columns represent different clusters

969 C) Pie chart showing the fractions of cells belonging to different clusters. The

970 fractions are pooled from two time points.

971 D) Graphical visualisation showing the cells taken into differential expression

972 analysis. Red shows the clonally expanded and mutated clonotype, and grey cells

973 represent the cells from other clonotypes with similar phenotype

974 E) Volcano plot showing differentially expressed genes between clonotype of

975 interest and cells from other clonotypes with similar phenotype

976 F) Gene Set Enrichment Analysis results from the differential expression analysis.

977 Shown here are four of eleven HALLMARK-categories enriched (FDR qval $<0.05$ ) to

978 clonotype of interest.

979

980 
$981 \quad$ Figure 5.

982

A

983

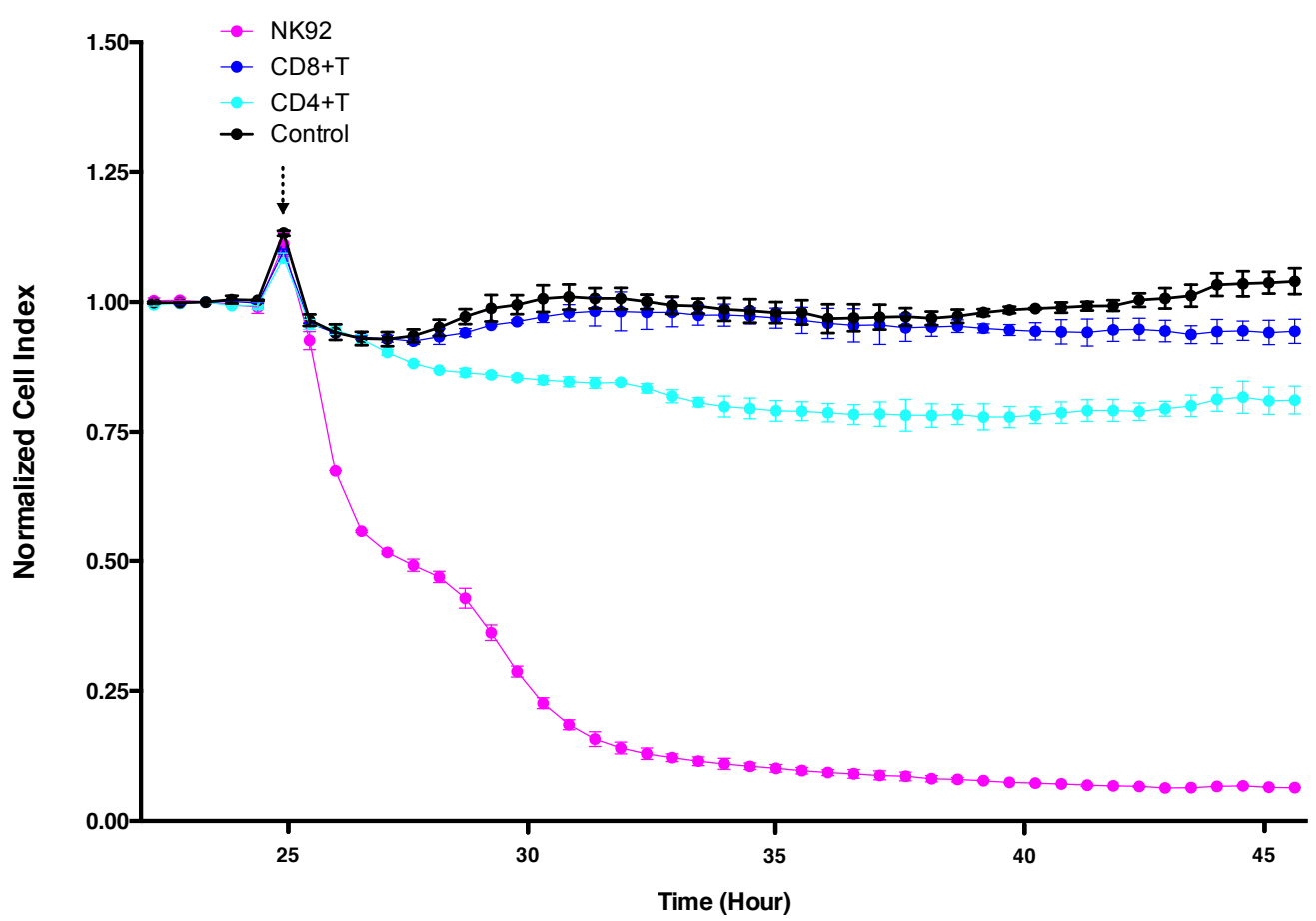

B

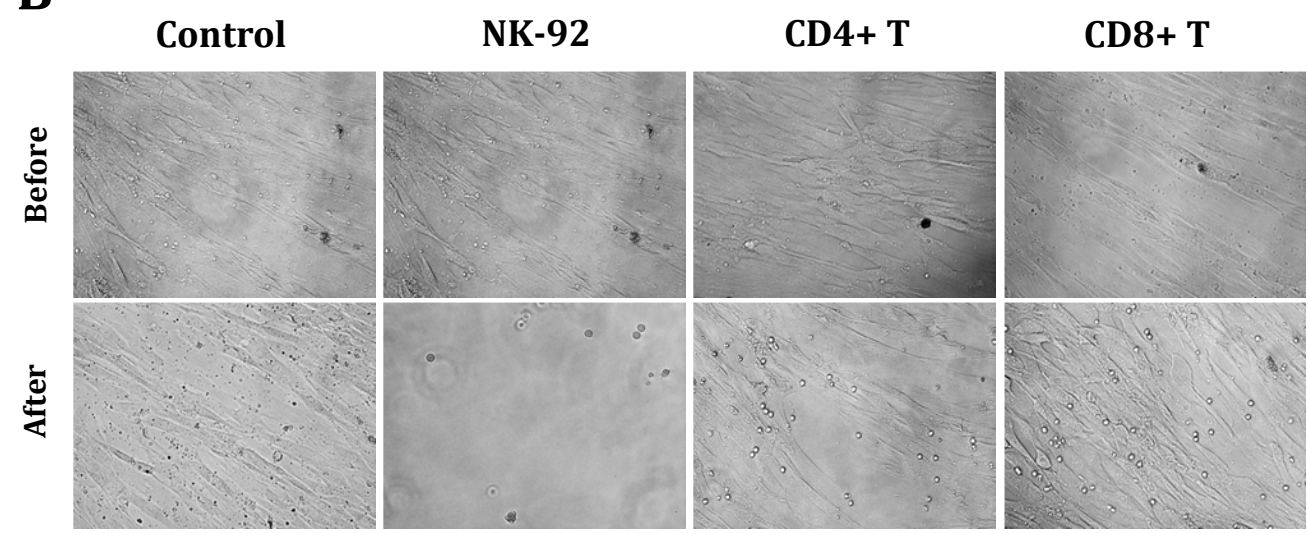


984 Figure 5. Real-time monitoring of cellular cytotoxicity by electrical impedance

985 measurement. Real-time cell analysing (RTCA) systems, xCELLigence $^{\mathrm{TM}}$, was applied

986 to monitor real-time killing effect of primary fibroblasts obtained from the index

987 patient.

988 A) The primary fibroblasts were cultured as monolayers for 24 hours to reach full

989 confluence. Once confluent, the effector cells; NK-92 cell line (positive control; pink

990 line), primary CD4+ T cells (light blue) and primary CD8+ T cells (dark blue) were

991 added to each well and co-cultured (Arrow). The control (black line) shows the

992 impedance of the fibroblasts without any added effectors. The cell impedance was

993 measured every 30 minutes for 48 hours. The measured impedance was expressed

994 as Cell Index with the normalization $(n=2)$. Data is representative of two

995 independent experiments showing similar results. The curve represents the mean

996 Cell Index value from 2 separate wells \pm SD.

997 B) Monolayers of the primary fibroblast were visualized before the addition of the

998 effector cells by inverted microscope (Nikon Eclipse TS100)(upper panels). In the end

999 of the experiment, each well was washed with PBS to photograph the live and still

1000 attached fibroblast. 
1002 A

1003

1004

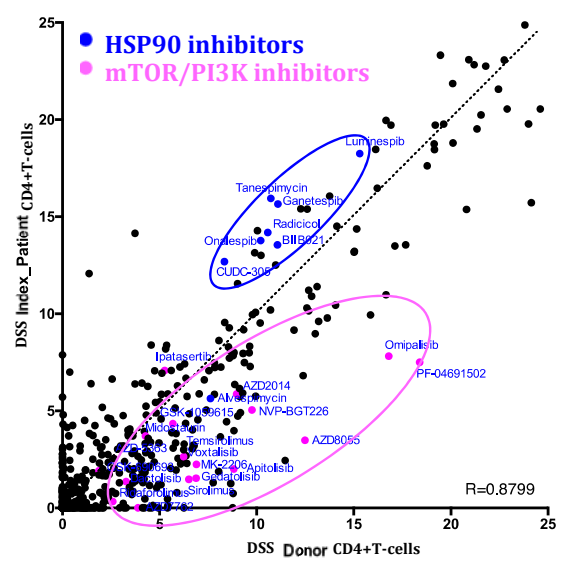

C

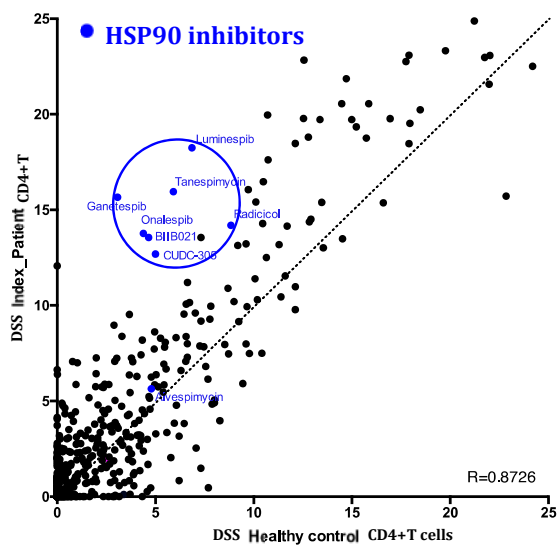

E

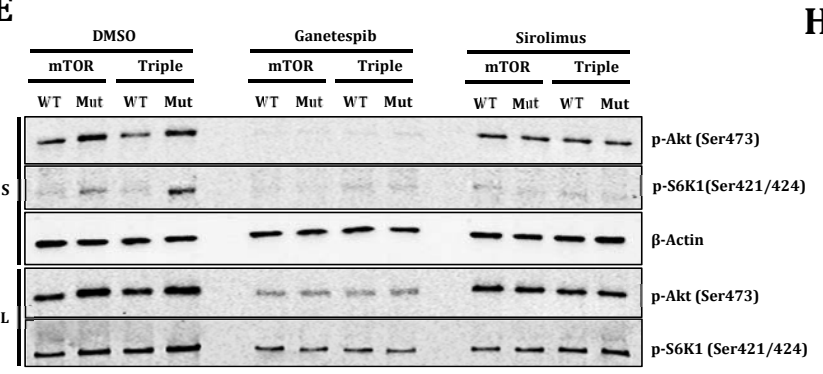

$\mathbf{F}$

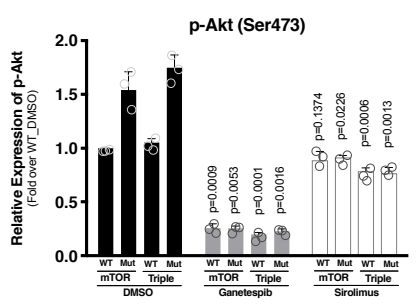

\section{G}

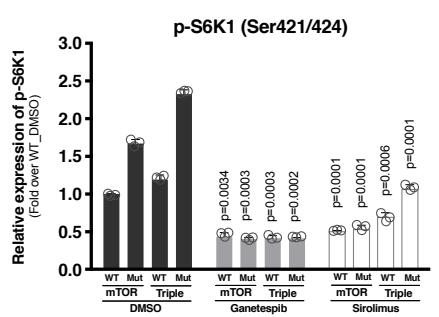

B
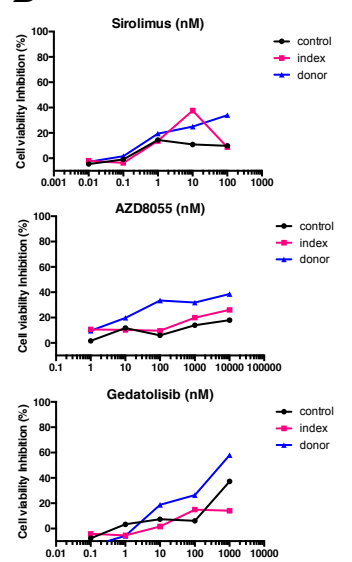

D
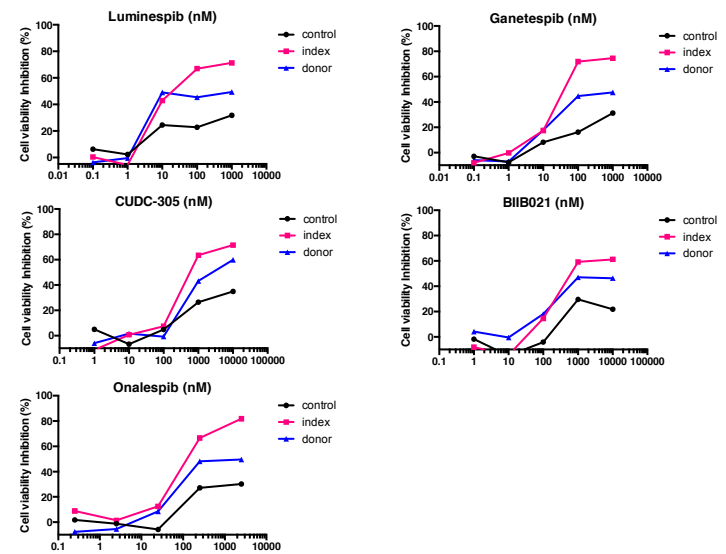

H

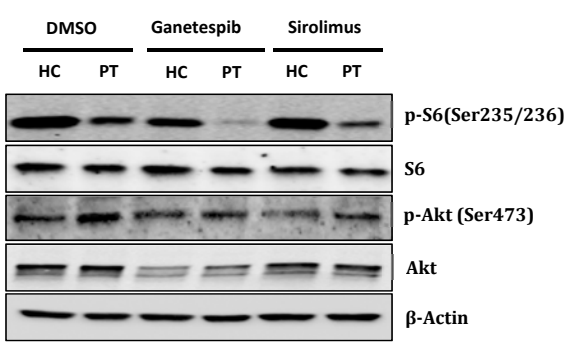

I

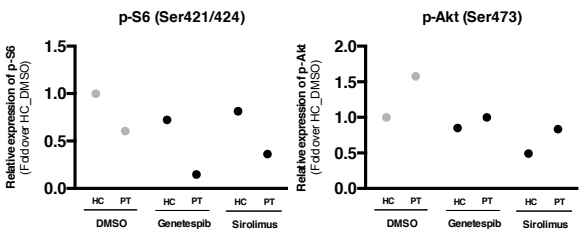


1005 Figure 6. Drug Sensitivity and Resistance testing (DSRT) of CD4+T cells from index

1006 patient compared with CD4+T cells from both healthy control and sibling donor.

1007 Ex vivo DSRT was performed on fresh CD4+T cells from index patient, donor, and

1008 healthy control. Correlation of drug sensitivity scores (DSS) indicating cell viability

1009 inhibition measured by CellTiter-Glo 2.0 (Promega, USA). DSS is a quantitative

1010 measurement of a drug response based on the area under the curve (AUC) with

1011 further normalization. Higher DSS denote better killing activity.

1012 A) Correlation of DSS scores between index patient and donor CD4+ T cells.

1013 B) Individual dose response curves of index patient, donor, and healthy control CD4+

1014 T cells for MTOR inhibitors

1015 C) Correlation of DSS scores between index patient and healthy control CD4+ T cells.

1016 D) Individual dose response curves of index patient, donor, and healthy control CD4+

1017 T cells for HSP90 inhibitors

1018 (E) Stably expressed MTOR wildtype (WT), mutant (P2229R), Triple (MTOR, TLR2 and

1019 NFKB2) wildtype (WT) and Triple mutant (MTOR P2229R, TLR2 W559L, and NFKB2

1020 P882Q) (Mut) in HEK293 cells were treated with HSP90 inhibitor (Ganetespib,

1021 100nM) or mTOR inhibitor (Sirolimus, 100nM) for 12 hours. Western blot analysis

1022 was performed with the use of anti-pAkt, anti-pS6K1, and anti- $\beta$-actin antibodies.

1023 Different amount of total protein was loaded in the upper panel (S, $30 \mathrm{ug}$ ) and the

1024 lower panel (L, 50 ug). Data is representative of three independent experiments.

1025 (F and G) Relative expression levels of phospho-Akt (F) and phospho-S6K1 (G) were

1026 estimated by measuring each band intensity using ImageJ software (Rasband, W.S.,

1027 ImageJ, U. S. National Institutes of Health, Bethesda, Maryland, USA,

1028 https://imagej.nih.gov/ij/, 1997-2016). Mean \pm SEM are shown ( $\mathrm{n}=3$ for group). $\mathrm{P}$ 
1029 values are derived from unpaired t-test with Welch's correction ( $p$ value on each bar

1030 indicates statistical significance between DMSO and Ganetespib/Sirolimus).

$1031 \quad(\mathrm{H})$ Isolated CD4+T cells from healthy control $(\mathrm{HC})$ and index patient $(\mathrm{PT})$ were

1032 treated with HSP90 inhibitor (Ganetespib, 100nM) or mTOR inhibitor (Sirolimus,

$1033100 \mathrm{nM}$ ) for 12 hours. Cells were lysed and proteins (25ug of total protein) were run

1034 on the SDS-PAGE gel. Western blot analysis was performed with the use of anti-S6,

1035 anti-pS6, anti-Akt, anti-pAkt and anti- $\beta$-actin antibodies.

1036 I) Relative expression levels of phospho-Akt and phospho-S6 were estimated by

1037 measuring band intensity with ImageJ software.

1038

1039 